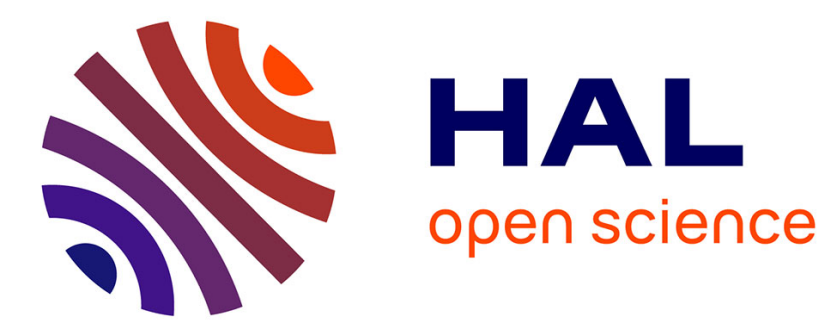

\title{
Building a class of fuzzy equivalence relations
}

\author{
Laurent Foulloy, Eric Benoit
}

\section{To cite this version:}

Laurent Foulloy, Eric Benoit. Building a class of fuzzy equivalence relations. Fuzzy Sets and Systems, 2006, 157 (157), pp.1417-1437. 10.1016/j.fss.2006.01.013 . hal-00428595

\section{HAL Id: hal-00428595 \\ https://hal.science/hal-00428595}

Submitted on 22 Oct 2019

HAL is a multi-disciplinary open access archive for the deposit and dissemination of scientific research documents, whether they are published or not. The documents may come from teaching and research institutions in France or abroad, or from public or private research centers.
L'archive ouverte pluridisciplinaire HAL, est destinée au dépôt et à la diffusion de documents scientifiques de niveau recherche, publiés ou non, émanant des établissements d'enseignement et de recherche français ou étrangers, des laboratoires publics ou privés. 


\title{
Building a Class of Fuzzy Equivalence Relations
}

\author{
Laurent Foulloy*, Eric Benoit \\ Université de Savoie, \\ LISTIC/ESIA \\ B.P. 806 ,
}

F-74016 Annecy cedex, France

\begin{abstract}
In this paper, we propose a practical method, given a strict triangular norm with a convex additive generator, for deriving a fuzzy equivalence relation whose reflexivity condition generalizes Ruspini's definition of fuzzy partitions. The properties of the relations, their comparison, their transitivity, the construction of fuzzy equivalence relations on cartesian products are presented. A large part of the paper is devoted to applications with fuzzy partitions defined on the real line. Several examples, including the fairy tale problem from De Cock and Kerre [12], the comparison of colored objects and comfort situations are proposed.
\end{abstract}

Keywords: Fuzzy equivalence relations, approximate equalities, strict t-norms, reflexivity, fuzzy meanings and descriptions, fuzzy partitions.

\section{Introduction}

In many applications, especially in fuzzy control, triangular or trapezoidal membership functions are used. Moreover, these membership functions define a fuzzy partition in the sense of Ruspini [25] as shown in figure 1.

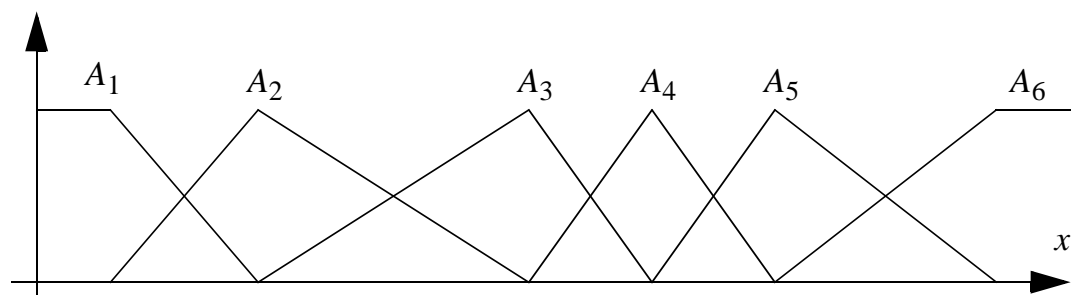

Fig. 1 Fuzzy partition in the sense of Ruspini.

It is often recognized that this type of membership functions are simple to handle and to compute with. Only a

*Corresponding author. Tel.: +(33) 450096 549; fax: +(33) 450096559.

E-mail address: Laurent.Foulloy@univ-savoie.fr 
few papers have tried to bring sound motivations for what can be considered as the simplest possible form for membership functions. Pedrycz proposed two main interpretations: one is based on equalization, like entropy equalization, while the second one relies on the error-free reconstruction of a fuzzified signal by means of a defuzzification interface [23], [24]. In [14], de Soto and Recasens, showed that fuzzy partitions based on triangular fuzzy numbers are obtained as compatible partitions associated with indistinguishability operators associated with the Lukasiewicz triangular norm. Dubois et al. also showed that triangular fuzzy numbers can be interpreted as the optimal probability-to-possibility transformation of a uniform probability distribution in a bounded interval [16].

It is also quite conventional to use sum-product inference in fuzzy systems, like in Mizumoto fuzzy controllers for instance. It is worth noticing that the sum operator is not a t-conorm. However, when a triangular based fuzzy partition is used, the bounded sum, that is Lukasiewicz t-conorm, reduces to the sum operator and the output of the fuzzy system also satisfies Ruspini’s condition on the cartesian product [2].

In this paper, we introduce a class of fuzzy equivalence relations whose reflexivity condition generalises Ruspini’s condition. Compatible fuzzy partitions enable to obtain various types of membership functions, including the triangular ones. In section 2, basic definitions are provided. In section 3 , it is shown in which conditions the class of relations which is studied in this paper becomes fuzzy equivalence relations. General properties are given, including the comparison between relations, their transitivity and the construction of fuzzy equivalence relations on cartesian products. A short discussion concludes this section. The links between fuzzy partitions and a linguistic approach are presented in section 4. Finally, several examples are proposed in section 5. The first one concerns the comparison between colored objects. The second one deals with the fairy-tale example proposed by De Cock and Kerre [12]. Then, fairy-tale characters with colored clothes are used to illustrate an equivalence relation on a cartesian product. Finally, the analysis of comfort situations is proposed to emphasize the construction of fuzzy partitions associated with fuzzy equivalence relations.

\section{Basic definitions}

Definition 1: Let $E$ be a set of vectors in $[0,1]^{n}$. Let $T$ be a t-norm. Let $R_{T}$ be the mapping from $E \times E$ to the positive real line, defined from the pair $(E, T)$, as :

$$
\forall(a, b) \in E^{2}, R_{T}(a, b)=\sum_{i=1}^{n} T\left(a_{i}, b_{i}\right) .
$$


Definition 2: The mapping $R_{T}$ is said to be:

i) reflexive on $E$ if $\forall a \in E, R_{T}(a, a)=1$.

ii) a fuzzy relation on $E$ if $\forall(a, b) \in E^{2}, R_{T}(a, b) \leq 1$.

iii) a reflexive fuzzy relation on $E$ if it is a fuzzy relation on $E$ and is reflexive on $E$.

iv) a symmetric fuzzy relation on $E$ if it is a fuzzy relation on $E$ and is symmetric, i.e. $\forall(a, b) \in E^{2}, R_{T}(a, b)=R_{T}(b, a)$.

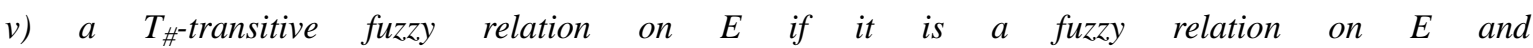
$\forall(a, b, c) \in E^{3}, T_{\#}\left(R_{T}(a, b), R_{T}(b, c)\right) \leq R_{T}(a, c)$.

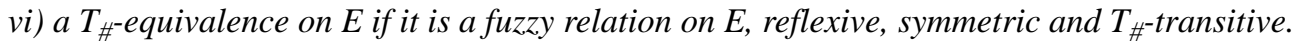

Definition 3: The mapping $\phi:[0,1] \rightarrow[0,1]$, defined by $\phi(u)=T(u, u)$, is called the diagonal section of the $t$ norm $T[18]$.

Remark 1: Let $T_{M}:[0,1]^{2} \rightarrow[0,1]$ be the t-norm defined by $T_{M}(u, v)=\min (u, v)$. The reflexivity condition for the mapping $R_{T_{M}}$ becomes:

$\forall a \in E, R_{T_{M}}(a, a)=\sum_{i=1}^{n} \phi\left(a_{i}\right)=\sum_{i=1}^{n} \min \left(a_{i}, a_{i}\right)=\sum_{i=1}^{n} a_{i}=1$.

This condition corresponds to Ruspini’s definition of a fuzzy partition [25].

Definition 4: Let $P=\left\{A_{i}\right\}_{i \in I}$ be a finite family of fuzzy sets of $X$, such that $\forall i \in I, A_{i} \neq \varnothing$, and $\phi$ be a $[0,1]$ automorphism. The family $P$ is a $\phi$-partition on $X$ [8], if it verifies: $\forall x \in X, \sum_{i \in I} \phi\left(A_{i}(x)\right)=1$.

Remark 2: Thus, the reflexivity condition of the relations $R_{T}$ can be related to De Baets's and Mesiar's definition of $\phi$-partitions, using $\phi(u)=T(u, u)$ as the [0,1]-automorphism with $T$ a strict t-norm. Let us recall that a t-norm $T$ is strict if it is continuous and strictly monotone, i.e. $T(u, v)<T(u, w)$ whenever $u>0$ and $v<w$ (see [18] for a large covering on t-norms). 


\section{Properties of $\boldsymbol{R}_{T}$ mappings}

\subsection{Generalities}

Let $T$ be a strict t-norm. It is well-known that strict t-norms are isomorphic to the product, that is there exists a strictly increasing bijection $\theta:[0,1] \rightarrow[0,1]$, such that, for all $(u, v) \in[0,1]^{2}$ :

$T(u, v)=\theta^{-1}(\theta(u) \cdot \theta(v))$.

Thus, we have for all $u \in[0,1]$ :

$\phi(u)=T(u, u)=\theta^{-1}\left(\theta^{2}(u)\right)$.

As the bijection $\theta$ is strictly increasing, the function $\phi$ is also a strictly increasing bijection. The inverse function $\phi^{-1}$ is defined, for all $u \in[0,1]$, by:

$\phi^{-1}(u)=\theta^{-1}(\sqrt{\theta(u)})$.

Remark 3: The bijection $\phi$ can also be defined from the additive generator of the strict t-norm $T$. If $T$ is a strict tnorm, it has an additive generator $t:[0,1] \rightarrow[0, \infty]$ which is a strictly continuous decreasing function such that $t(0)=\infty, t(1)=0$ and:

$\forall(u, v) \in[0,1]^{2}, T(u, v)=t^{-1}(t(u)+t(v))$.

Therefore, it can easily be deduced, for all $u \in[0,1]$ :

$\phi(u)=t^{-1}(2 t(u))$,

$\phi^{-1}(u)=t^{-1}\left(\frac{t(u)}{2}\right)$.

Remark 4: It is always simple to build a set $E$ of vectors in $[0,1]^{n}$ such that the mapping $R_{T_{M}}$ is reflexive on $E$. Equation (5) provides a simple means to define a set $F$ such that, given a strict t-norm $T$ with a diagonal section $\phi$, the mapping $R_{T}$ is reflexive on $F$. Indeed, it can be defined as follows:

$F=\left\{b \in[0,1]^{n} ; \exists a \in E\right.$ such that $b_{i}=\phi^{-1}\left(a_{i}\right)$, for all $\left.i \in\{1, \ldots, n\}\right\}$.

Obviously, we have: 


$$
\forall b \in F, R_{T}(b, b)=\sum_{i=1}^{n} \phi\left(b_{i}\right)=\sum_{i=1}^{n} \phi\left(\phi^{-1}\left(a_{i}\right)\right)=\sum_{i=1}^{n} a_{i}=1
$$

This property will be used in section 4 to define fuzzy partitions on $\Re$.

Example 1: Let $E=\left\{a, a^{\prime}\right\}$ with $a=\left[\begin{array}{lll}0.3 & 0.2 & 0.5\end{array}\right]$ and $a^{\prime}=\left[\begin{array}{lll}0.1 & 0.7 & 0.2\end{array}\right]$. The mapping $R_{T_{M}}$, defined from the pair ( $E, T_{M}$ ), is a fuzzy reflexive relation on $E$.

\begin{tabular}{|c|c|c|}
\hline$R_{T_{M}}$ & $a$ & $a^{\prime}$ \\
\hline$a$ & 1.000 & 0.500 \\
\hline$a^{\prime}$ & 0.500 & 1.000 \\
\hline
\end{tabular}

Table 1: Fuzzy reflexive relation on $E$.

Example 2: Let $T_{P}:[0,1]^{2} \rightarrow[0,1]$ be the t-norm defined by $T_{P}(u, v)=u$.v. According to equation (10), we get $F=\left\{b, b^{\prime}\right\}$ with $b=\left[\begin{array}{lll}0.5477 & 0.44720 .7071\end{array}\right]$ and $b^{\prime}=\left[\begin{array}{lll}0.3162 & 0.83670 .4472\end{array}\right]$. The mapping $R_{T_{P}}$, defined from the pair $\left(E, T_{P}\right)$, is also a fuzzy reflexive relation on $F$.

\begin{tabular}{|c|l|l|}
\hline$R_{T_{P}}$ & \multicolumn{1}{|c|}{$b$} & \multicolumn{1}{c|}{$b^{\prime}$} \\
\hline$b$ & 1.000 & 0.8636 \\
\hline$b^{\prime}$ & 0.8636 & 1.000 \\
\hline
\end{tabular}

Table 2: Fuzzy reflexive relation on $F$.

Proposition 1: All strict t-norms T satisfy:

$T(u, v)=\phi^{-1}(T(\phi(u), \phi(v)))$.

Proof. Let us write the t-norm using its multiplicative generator.

$$
T(\phi(u), \phi(v))=\theta^{-1}(\theta(\phi(u)) \cdot \theta(\phi(v))) .
$$

Because, $\theta(\phi(u))=\theta^{2}(u)$, we have:

$$
T(\phi(u), \phi(v))=\theta^{-1}\left(\theta^{2}(u) \cdot \theta^{2}(v)\right)=\phi(T(u, v)) .
$$


Proposition 2: Let $T$ be a strict t-norm. Let $E$ be a set of vectors in $[0,1]^{n}$. Let $R_{T}$ be the mapping reflexive on $E$.

If the additive generator $t$ of the t-norm $T$ is strictly convex, then the following are equivalent for all $(a, b) \in E^{2}$ :

(i) $\exists i \in\{1, \ldots, n\}, a_{i} \neq b_{i}$,

(ii) $\quad R_{T}(a, b)<1$,

and, therefore, the relation $R_{T}$ is a reflexive fuzzy relation.

Proof. (ii) implies (i) from the fuzzy reflexivity condition of $R_{T}$.

To prove that (i) implies (ii), let us remark that if the additive generator $t$ of the t-norm $T$ is strictly convex then, as it is decreasing by definition, its inverse $t^{-1}$ is also strictly convex. Therefore, for all $a_{i} \neq b_{i}$ :

$$
\begin{aligned}
& 2 t^{-1}\left(t\left(a_{i}\right)+t\left(b_{i}\right)\right)<t^{-1}\left(2 t\left(a_{i}\right)\right)+t^{-1}\left(2 t\left(b_{i}\right)\right) \\
& \Leftrightarrow 2 T\left(a_{i}, b_{i}\right)<T\left(a_{i}, a_{i}\right)+T\left(b_{i}, b_{i}\right) .
\end{aligned}
$$

Due to the fuzzy reflexivity condition of $R_{T}$, we have $R_{T}(a, a)=1$ and $R_{T}(b, b)=1$, leading to:

$$
\begin{aligned}
& 2 \sum_{i=1}^{n} T\left(a_{i}, b_{i}\right)<\sum_{i=1}^{n} T\left(a_{i}, a_{i}\right)+\sum_{i=1}^{n} T\left(b_{i}, b_{i}\right) \\
& \Leftrightarrow 2 R_{T}(a, b)<R_{T}(a, a)+R_{T}(b, b) \\
& \Leftrightarrow R_{T}(a, b)<1 .
\end{aligned}
$$

Remark 5: This property is satisfied for the following families because their additive generators are strictly convex for the given parameter ranges:

- $T_{\lambda}{ }^{F}$ of Frank t-norms when $\left.\lambda \in\right] 0, \infty[$,

- $T_{\lambda}{ }^{\text {SS }}$ of Schweizer-Sklar t-norms when $\left.\lambda \in\right]-\infty, 1[$,

- $T_{\lambda}{ }^{A A}$ of Aczél-Alsina t-norms when $\lambda \in[1, \infty[$,

- $T_{\lambda}{ }^{D}$ of Dombit-norms when $\lambda \in[1, \infty[$,

- $T_{\lambda}^{H}$ of Hamacher t-norms when $\lambda \in[0,2[$.

The family of Frank t-norms is continuous with respect to the parameter $\lambda$ and we have $T_{0}{ }^{F}=T_{M}$ where $T_{M}$ 
is the minimum. Although $T_{M}$ is not a strict t-norm, proposition 2 holds true for this t-norm (Let us note that the proof without using the continuity of a family of t-norms is quite obvious). It can also easily be shown that all tnorms built as the ordinal sum [18] of strict t-norms with convex additive generators also satisfy the equivalence in proposition 2.

\subsection{Comparison of $R_{T}$ fuzzy relations}

In this section, after a general proposition, it will be shown that $R_{T}$ fuzzy relations can be compared with $R_{T_{M}}$ relations. Then, given two strict t-norms $T_{1}$ and $T_{2}$, the condition under which the relation $R_{T_{1}}$ can be compared with $R_{T_{2}}$ is given.

Proposition 3: Let $T$ be a strict t-norm. Let $t$ be its additive generator and $\phi$ its diagonal section. For any $(u, v) \in[0,1]^{2}$ and $u^{\prime}=\phi^{-1}(u)$ and $v^{\prime}=\phi^{-1}(v)$, we have:

$T\left(u^{\prime}, v^{\prime}\right)=t^{-1}\left(\frac{t(u)+t(v)}{2}\right)$.

Proof. Let us write $T$ with its additive generator, that is $T\left(u^{\prime}, v^{\prime}\right)=t^{-1}\left(t\left(u^{\prime}\right)+t\left(v^{\prime}\right)\right)$ and use equation (8) in remark 3, that is $\phi^{-1}(u)=t^{-1}\left(\frac{t(u)}{2}\right)$. Replacing into the definition of the t-norm, we get:

$$
T\left(u^{\prime}, v^{\prime}\right)=t^{-1}\left(t\left(t^{-1}\left(\frac{t(u)}{2}\right)\right)+t\left(t^{-1}\left(\frac{t(v)}{2}\right)\right)\right)=t^{-1}\left(\frac{t(u)+t(v)}{2}\right) .
$$

Proposition 4: Let T be a strict t-norm whose additive generator t is strictly convex and diagonal section is denoted

$\phi$. Let $E$ be a set of vectors in $[0,1]^{n}$. Let $R_{T}$ be the mapping reflexive on $E$. Then, for all $(a, b) \in E^{2}$, $R_{T}(a, b) \geq R_{T_{M}}\left(a^{\prime}, b^{\prime}\right)$ where $a_{i}^{\prime}=\phi\left(a_{i}\right)$ and $b_{i}^{\prime}=\phi\left(b_{i}\right)$ for all $i \in\{1, \ldots, n\}$.

Proof. If $a^{\prime}{ }_{i} \leq b^{\prime}{ }_{i}$ then, because $t$ is a strictly decreasing function, $a^{\prime}{ }_{i} \leq t^{-1}\left(\frac{t\left(a_{i}^{\prime}\right)+t\left(b_{i}^{\prime}\right)}{2}\right) \leq b_{i}^{\prime}$ and, therefore, $\min \left(a_{i}^{\prime}, b_{i}^{\prime}\right) \leq t^{-1}\left(\frac{t\left(a_{i}^{\prime}\right)+t\left(b_{i}^{\prime}\right)}{2}\right)$. Then, using proposition 3 with $u=\phi\left(a_{i}\right)$ and $v=\phi\left(b_{i}\right)$ leads to 
$\min \left(a_{i}^{\prime}, b_{i}^{\prime}\right) \leq T\left(a_{i}, b_{i}\right)$, which holds true for all $i \in\{1, \ldots, n\}$. Thus, we have $R_{T}(a, b) \geq R_{T_{M}}\left(a^{\prime}, b^{\prime}\right)$.

Proposition 5: Let $T_{1}$ and $T_{2}$ be two strict $t$-norms whose respective additive generators $t_{1}$ and $t_{2}$ are strictly convex and diagonal sections are respectively denoted $\phi_{1}$ and $\phi_{2}$. Let E and F be two sets of vectors in $[0,1]^{n}$. Let $R_{T_{1}}$ and $R_{T_{2}}$ be two mappings respectively reflexive on $E$ and $F$. If the additive generators $t_{1}$ and $t_{2}$ are such that $t_{1} \mathrm{o} t_{2}^{-1}$ is concave then, for all $(a, b) \in E^{2}, R_{T_{1}}(a, b) \geq R_{T_{2}}\left(a^{\prime}, b^{\prime}\right)$ where $\left(a^{\prime}, b^{\prime}\right) \in F^{2}, a^{\prime}{ }_{i}=\phi_{2}^{-1}\left(\phi_{1}\left(a_{i}\right)\right)$ and $b_{i}^{\prime}=\phi_{2}^{-1}\left(\phi_{1}\left(b_{i}\right)\right)$ for all $i \in\{1, \ldots, n\}$

Proof. If $t_{1}$ o $t_{2}^{-1}$ is concave, then for all $(x, y) \in[0, \infty]^{2}$,

$t_{1} \circ t_{2}^{-1}\left(\frac{x+y}{2}\right) \geq \frac{t_{1} \circ t_{2}^{-1}(x)+t_{1} \circ t_{2}^{-1}(y)}{2}$

Because $t_{2}$ is bijective, there exists $(u, v) \in[0,1]^{2}$ such that $x=t_{2}(u)$ and $y=t_{2}(v)$. Replacing in equation (16) leads to:

$t_{1} \mathrm{o} t_{2}^{-1}\left(\frac{t_{2}(u)+t_{2}(v)}{2}\right) \geq \frac{t_{1}(u)+t_{1}(v)}{2}$

Composing by $t_{1}^{-1}$ and taking into account that $t_{1}^{-1}$ is strictly decreasing gives:

$t_{2}^{-1}\left(\frac{t_{2}(u)+t_{2}(v)}{2}\right) \leq t_{1}^{-1}\left(\frac{t_{1}(u)+t_{1}(v)}{2}\right)$

Now, using proposition 3 with $u=\phi_{1}\left(a_{i}\right)=\phi_{2}\left(a^{\prime}{ }_{i}\right)$ and $v=\phi_{1}\left(b_{i}\right)=\phi_{2}\left(b^{\prime}{ }_{i}\right)$, it comes:

$T_{2}\left(a^{\prime}, b_{i}^{\prime}\right) \leq T_{1}\left(a_{i}, b_{i}\right)$

Finally, since (19) holds for all $i \in(1, \ldots, n)$, we get:

$\sum_{i=1}^{n} T_{1}\left(a_{i}, b_{i}\right) \geq \sum_{i=1}^{n} T_{2}\left(a^{\prime}, b^{\prime}{ }_{i}\right)$ and, therefore, $R_{T_{1}}(a, b) \geq R_{T_{2}}\left(a^{\prime}, b^{\prime}\right)$.

Corollary 1: If $t_{1}$ o $t_{2}^{-1}$ is concave then $T_{1} \leq T_{2}$ and $R_{T_{2}}\left(a^{\prime}, b^{\prime}\right) \leq R_{T_{1}}(a, b)$. 
Proof. From the work of Schweizer and Sklar, it is known that, for continuous Archimedian t-norms, $t_{1} \mathrm{o} t_{2}^{-1}$ is subadditive and $T_{1} \leq T_{2}$ are equivalent [18]. As a corollary, if $t_{1}$ o $t_{2}^{-1}$ is concave then it is subadditive [18]. Thus, if $t_{1}$ o $t_{2}^{-1}$ is concave then $T_{1} \leq T_{2}$ and, according to proposition $5, R_{T_{2}}\left(a^{\prime}, b^{\prime}\right) \leq R_{T_{1}}(a, b)$.

\subsection{Transitivity of $\boldsymbol{R}_{T}$ fuzzy relations}

In this section, we first demonstrate that all $R_{T}$ fuzzy relations are $T_{D}$-transitive, where $T_{D}$ is the smallest tnorm. Although this theorem is general, it also quite weak since many reflexive relations are $T_{D^{-}}$transitive. Then, a second proposition shows that $R_{T_{M}}$ relations are $T_{L}$-transitive, where $T_{L}$ is the Lukasiewicz t-norm. Another proof, based on the equality $\min (u, v)=\frac{u+v-|u-v|}{2}$, was proposed in by Bezdek and Harris [3]. A third proposition concerning the transitivity of $R_{T_{P}}$ relations is given. Finally, simulation results are proposed for the family of Frank t-norms.

Proposition 6: Let $T$ be a strict t-norm. Let $E$ be a set of vectors in $[0,1]^{n}$. Let $R_{T}$ be the relation reflexive on $E$. If the additive generator of the t-norm $T$ is strictly convex, then $R_{T}$ is a fuzzy relation $T_{D}$-transitive on $E$ where $T_{D}:[0,1]^{2} \rightarrow[0,1]$ is the smallest t-norm, that is:

$T_{D}(x, y)=0$ if $x \neq 1$ and $y \neq 1$,

$T_{D}(x, y)=\min (x, y)$ otherwise

Proof. According to proposition 2, $R_{T}$ is a fuzzy relation. Now, we have to show:

$\forall(a, b, c) \in E^{3}, T_{D}\left(R_{T}(a, b), R_{T}(b, c)\right) \leq R_{T}(a, c)$.

Three cases must be considered:

i) $R_{T}(a, b)=1$ and $R_{T}(b, c)=1$. In this case, according to proposition 2 and the reflexivity of $R_{T}$, we have $a=b=c$, therefore equation (21) holds true.

ii) $R_{T}(a, b)=1$ or $R_{T}(b, c)=1$. According to the symmetry of $T_{D}$, we will only consider the case where $R_{T}(a, b)=1$. The left hand side part of equation (21) is equal to $R_{T}(b, c)$. According to proposition 2 and the 
reflexivity of $R_{T}$, we have $a=b$. Thus, equation (21) holds true.

iii) $R_{T}(a, b) \neq 1$ and $R_{T}(b, c) \neq 1$. In this case equation (21) is always satisfied since the left hand side part is equal to 0 .

Remark 6: Under the assumptions of proposition 6, all relations $R_{T}$ on $E$ are $T_{D}$-equivalences on $E$.

Proposition 7: Let $T=T_{M}$ where $T_{M}$ is the minimum. Let $E$ be a set of vectors in $[0,1]^{n}$. Let $R_{T_{M}}$ be the reflexive

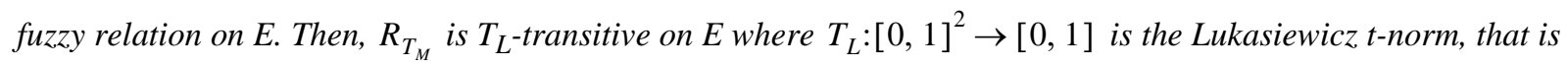
$T_{L}(u, v)=\max (u+v-1,0)$.

Proof. We have to show:

$\forall(a, b, c) \in E^{3}, \max \left(R_{T_{M}}(a, b)+R_{T_{M}}(b, c)-1,0\right) \leq R_{T_{M}}(a, c)$.

Let us replace $R_{T_{M}}$ by its definition:

$$
\begin{aligned}
& \max \left(\sum_{i=1}^{n}\left(T_{M}\left(a_{i}, b_{i}\right)+T_{M}\left(b_{i}, c_{i}\right)\right)-1,0\right) \leq \sum_{i=1}^{n} T_{M}\left(a_{i}, c_{i}\right) . \\
& \quad \text { If } \sum_{i=1}^{n}\left(T_{M}\left(a_{i}, b_{i}\right)+T_{M}\left(b_{i}, c_{i}\right)\right)-1 \leq 0, \text { Eq. (23) always holds true. }
\end{aligned}
$$

$n$

In the other case, the reflexivity of the relation gives $\sum_{i=1} b_{i}=1$ which can be replaced in Eq. (23) leading to:

$\sum_{i=1}^{n}\left(T_{M}\left(a_{i}, b_{i}\right)+T_{M}\left(b_{i}, c_{i}\right)\right) \leq \sum_{i=1}^{\prime \prime}\left(b_{i}+T_{M}\left(a_{i}, c_{i}\right)\right)$

Using the distributivity of the addition with respect to the minimum, we have:

$$
\sum_{i=1}^{n}\left(T_{M}\left(a_{i}, b_{i}\right)+T_{M}\left(b_{i}, c_{i}\right)\right) \leq \sum_{i=1}^{n} T_{M}\left(a_{i}+b_{i}, c_{i}+b_{i}\right)
$$

which is always true. 
Proposition 8: Let $T=T_{P}$ where $T_{P}$ is the product. Let $E$ be a set of vectors in $[0,1]^{n}$. Let $R_{T_{P}}$ be the reflexive fuzzy relation on $E$. Then, $R_{T_{P}}$ is $T_{0.5}^{Y}$-transitive on $E$ where $T_{\lambda}^{Y}:[0,1]^{2} \rightarrow[0,1]$ is Yager's t-norm, that is $\left\{\begin{array}{l}T_{D}(u, v), \text { if } \lambda=0, \\ \max \left(1-\left((1-u)^{\lambda}+(1-v)^{\lambda}\right)^{\frac{1}{\lambda}}, 0\right), \text { otherwise. }\end{array}\right.$

Proof. We have to show:

$$
\begin{gathered}
\forall(a, b, c) \in E^{3}, \max \left(1-\left(\sqrt{1-R_{T_{P}}(a, b)}+\sqrt{1-R_{T_{P}}(b, c)}\right)^{2}, 0\right) \leq R_{T_{P}}(a, c) . \\
\text { If }\left(\sqrt{1-R_{T_{P}}(a, b)}+\sqrt{1-R_{T_{P}}(b, c)}\right)^{2}>1 \text {, Eq. (26) always holds true. }
\end{gathered}
$$

In the other case, according to equation (1), $R_{T_{P}}(a, b)$ is the dot product of the vectors $a$ and $b$. Thus, we have to show:

$$
\sqrt{1-a \cdot b}+\sqrt{1-b \cdot c} \geq \sqrt{1-a \cdot c}
$$

Let us note that the vectors in $E$ are unit vectors due to the reflexivity condition of the fuzzy relation $R_{T_{P}}$. Let us denote respectively $\overrightarrow{O A}, \overrightarrow{O B}, \overrightarrow{O C}$ the three vectors $a, b$, and $c$. The norm of the vector $\overrightarrow{A B}$ is given by:

$$
\|\overrightarrow{A B}\|=\sqrt{\sum_{i=1}^{n}\left(b_{i}-a_{i}\right)^{2}}=\sqrt{\sum_{i=1}^{n}\left(a_{i}^{2}+b_{i}^{2}-2 a_{i} b_{i}\right)}=\sqrt{2} \sqrt{1-a \cdot b} .
$$

According to the triangular inequality, we have $\|\overrightarrow{A B}\|+\|\overrightarrow{B C}\| \geq\|\overrightarrow{A C}\|$ and therefore (27) holds true.

Remark 7: The t-norm $T_{L}$ and the family $T_{\lambda}^{Y}$ belong to the family of nilpotent t-norms. A t-norm $T_{\#}$ is nilpotent if it is continuous and each $a \in] 0,1[$ is a nilpotent element, i.e. there exists $n \in \mathbb{N}$ such that $a_{T}^{(n)}=T_{\#} \frac{(a, a, \ldots, a)}{n \text { times }}=0$. Let us also note that $T_{D}=T_{0}^{Y}$ because the family of Yager t-norms is continuous with respect to its parameter and strictly increasing. It is known that any nilpotent t-norm $T_{\#}$ is isomorphic to Lukasiewicz t-norm, i.e. there exists a strictly increasing bijection $\varphi:[0,1] \rightarrow[0,1]$ such that for all 
$(u, v) \in[0,1]^{2}:$

$T_{\#}(u, v)=\varphi^{-1}\left(T_{L}(\varphi(u), \varphi(v))\right)=\varphi^{-1}(\max (\varphi(u)+\varphi(v)-1,0))$.

This remark opens an interesting question. Given a set $E$ of vectors in $[0,1]^{n}$ and a t-norm $T$ with a strictly convex additive generator, can we find the greatest $T_{\#}$ nilpotent t-norm such that the relation $R_{T}$ reflexive on $E$ is a $T_{\#}$ equivalence on $E$ ?

As a first step towards an answer, figure 2 represents the highest $\lambda$, obtained by numerical computations, for which the relation $R_{T_{\alpha}{ }^{F}}$ associated with the family of Frank t-norms is $T_{\lambda}^{Y}$-transitive on $E$. The $x$-axis is the base 10 logarithm of Frank’s t-norm parameter $\alpha$.

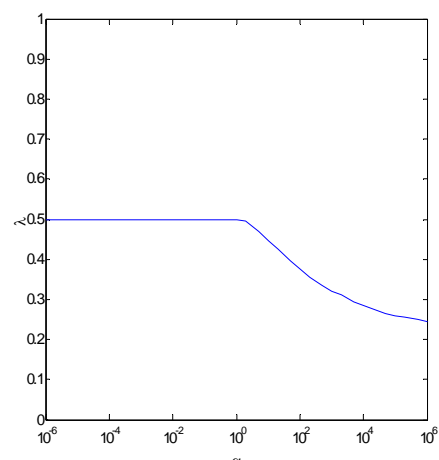

Fig. $2 \quad T_{\lambda}^{Y}$-transitivity of the family of Frank t-norms.

\subsection{Fuzzy equivalence on $E_{1} \times E_{2}$}

Equivalence relations are closely related to pseudo-metrics. In particular, if $Q$ is a $T_{\#}$-equivalence on $E$ then $d_{Q}: E^{2} \rightarrow[0, \infty]$ defined by $d_{Q}=t_{\# 0} Q$, with $t_{\#}$ the additive generator of $T_{\#}$, is a pseudo-metric on $E$ [9]. In this

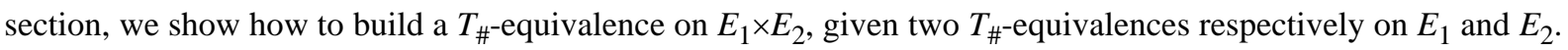
Thus, it makes it possible to keep the same pseudo-metric on the cartesian product.

Proposition 9: Let $T$ be a strict t-norm with a strictly convex additive generator and a diagonal section $\phi$. Let $E_{1}$ and $E_{2}$ be two sets of vectors respectively in $[0,1]^{n}$ and $[0,1]^{m}$. Let $R_{T}^{1}$ and $R_{T}^{2}$ be the two $T_{\# \text {-equivalences re- }}$ spectively on $E_{1}$ and $E_{2}$. Let $T^{*}:[0,1]^{2} \rightarrow[0,1]$ be the strict $t$-norm defined by:

$T^{*}(u, v)=\phi^{-1}(\phi(u) \cdot \phi(v))$.

Then, for all $\left(a, a^{\prime}\right) \in E_{1}^{2}$ and $\left(b, b^{\prime}\right) \in E_{2}^{2}$, the mapping $R_{T}$ defined by: 
$R_{T}\left((a, b),\left(a^{\prime}, b^{\prime}\right)\right)=\sum_{i=1 j=1}^{n} \sum_{i}^{m} T\left(T^{*}\left(a_{i}, b_{j}\right), T^{*}\left(a_{i}^{\prime}, b_{j}^{\prime}\right)\right)$,

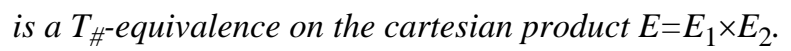

Proof. First of all, let us note that $T^{*}$ is a strict t-norm because, as shown in section $3, \phi$ is a strictly increasing bijection from $[0,1]$ to $[0,1]$, which can therefore be used as a multiplicative generator. Now, to prove the proposition, as the additive generator of the t-norm $T$ is strictly convex, it is sufficient to prove that the fuzzy relation $R_{T}$ is reflexive on $E$. The reflexivity of the relation $R_{T}$ is given, for all $(a, b) \in E^{2}$, by:

$$
R_{T}((a, b),(a, b))=\sum_{i=1 j=1}^{n} \sum_{j}^{m} \phi\left(T^{*}\left(a_{i}, b_{j}\right)\right)
$$

Replacing $T^{*}$ by its definition, then using the distributivity of the sum with respect to the product and, finally, the reflexivity of the relations $R_{T}^{1}$ and $R_{T}^{2}$ respectively on $E_{1}$ and $E_{2}$, we have:

$$
R_{T}((a, b),(a, b))=\sum_{i=1 j=1}^{n} \sum_{j=1}^{m} \phi\left(a_{i}\right) \cdot \phi\left(b_{j}\right)=\sum_{i=1}^{n} \phi\left(a_{i}\right) \cdot \sum_{j=1}^{m} \phi\left(b_{j}\right)=1
$$

Example 3: Let $E_{1}=\left\{a, a^{\prime}\right\}$ with $a=\left[\begin{array}{llll}0.36 & 0.48 & 0.80\end{array}\right]$ and $a^{\prime}=\left[\begin{array}{lll}0.48 & 0.60 & 0.64\end{array}\right]$ and $R_{T_{P}}^{1}$ be the relation on $E_{1}$. Let $E_{2}=\left\{b, b^{\prime}\right\}$ with $b=[0.280 .96]$ and $b^{\prime}=[0.600 .80]$ and $R_{T_{P}}^{2}$ be the relation on $E_{2}$. The resulting relations are:

\begin{tabular}{|c|c|c|}
\hline$R_{T_{P}}^{1}$ & $a$ & $a^{\prime}$ \\
\hline$a$ & 1.0000 & 0.9728 \\
\hline$a^{\prime}$ & 0.9728 & 1.0000 \\
\hline
\end{tabular}

\begin{tabular}{|c|c|c|}
\hline$R_{T_{P}}^{2}$ & $b$ & $b^{\prime}$ \\
\hline$b$ & 1.0000 & 0.9360 \\
\hline$b^{\prime}$ & 0.9360 & 1.0000 \\
\hline
\end{tabular}

\begin{tabular}{|c|c|c|c|c|}
\hline$R_{T_{P}}$ & $(a, b)$ & $\left(a^{\prime}, b\right)$ & $\left(a, b^{\prime}\right)$ & $\left(a^{\prime}, b^{\prime}\right)$ \\
\hline$(a, b)$ & 1.0000 & 0.9360 & 0.9728 & 0.9105 \\
\hline$\left(a^{\prime}, b\right)$ & 0.9360 & 1.0000 & 0.9105 & 0.9728 \\
\hline$\left(a, b^{\prime}\right)$ & 0.9728 & 0.9105 & 1.0000 & 0.9360 \\
\hline$\left(a^{\prime}, b^{\prime}\right)$ & 0.9105 & 0.9728 & 0.9360 & 1.0000 \\
\hline
\end{tabular}

Table 3: Fuzzy relations on $E_{1}, E_{2}$ and $E_{1} \times E_{2}$.

\subsection{Discussion}

It is well known that, given a crisp partition $P$ of a crisp set $X$, there exists a unique equivalence relation $Q$ such that $P$ is the quotient set of $X$ by this relation which is defined by:

$$
x Q y \Leftrightarrow \exists A \in P, x \in A \wedge y \in A .
$$


Now, let $I=\{1, \ldots, n\}$ and let us assume that the partition $P$ is a finite family of non-empty fuzzy sets, that is $P=\left\{A_{i}\right\}_{i \in I}$ and $\forall i \in I, A_{i} \neq \varnothing$, the fuzzy version of Eq. (32) is given by:

$\forall(x, y) \in X \times X, Q(x, y)=\sup _{i \in I} T\left(A_{i}(x), A_{i}(y)\right)$.

Since $I$ is finite, the supremum can be replaced by the maximum which itself can be replaced by a t-conorm $S$, leading to $\mathrm{Q}_{S-T}$ fuzzy relations defined by:

$\forall(x, y) \in X \times X, Q_{S-T}(x, y)=S_{i \in I} T\left(A_{i}(x), A_{i}(y)\right)$.

Given $x \in X$, let us denote $D_{x}$ the vector in $[0,1]^{n}$ and $D_{x}(i)$, its component defined by:

$\forall i \in I, \forall x \in X, D_{x}(i)=A_{i}(x)$

Thus, under the condition of proposition 2, we can link the fuzzy relations $Q_{S_{L}-T}$ with the fuzzy equivalence relations $R_{T}$, where $S_{L}:[0,1]^{2} \rightarrow[0,1]$ is Lukasiewicz triangular conorm, i.e. $S_{L}(u, v)=\min (u+v, 1)$, as follows:

$$
\begin{aligned}
& \forall(x, y) \in X \times X, Q_{S_{L}-T}(x, y)=S_{L}{ }_{i \in I} T\left(A_{i}(x), A_{i}(y)\right)=S_{L} \underset{i \in I}{ } T\left(D_{x}(i), D_{y}(i)\right)=\min \left(\sum_{i \in I} T\left(D_{x}(i), D_{y}(i)\right), 1\right) \\
& =\min \left(R_{T}\left(D_{x}, D_{y}\right), 1\right)=R_{T}\left(D_{x}, D_{y}\right) .
\end{aligned}
$$

This approach, which provides a restrictive class of equivalence relations (see [6] for a survey on fuzzy equivalence relations), relies on the same trends as the pioneering works of Bezdek and Harris on likeness relations [3] or Ovchinnikov's on proximity relations [22], which emphasize the definition of fuzzy partitions and study the properties of the associated relations. For example, a $R_{T_{M}}$ fuzzy relation is given in [3] for the Fuzzy CMeans clustering algorithm, where the $T_{L}$-transitivity is shown from the triangle inequality. Indeed, the reflexivity condition gives the constraint $\sum_{i=1} u_{i}(x)=1$ where $c$ is the number of classes and $u_{i}(x)$ the class membership function of the data set $X$.

As already mentioned, it is also closely related to $\phi$-partitions obtained from an algebraic (or strict) fuzzy partition, as proposed by De Baets and Mesiar [8]. Links between a linguistic variable and indistinguishability relations, introduced by Valverde and Trillas [26], [27], have been studied by De Soto and Recasens [14].

Finally, let us also remark that $Q_{S_{L}-T}$ fuzzy relations are a particular case of the parametrized family $Q_{S_{\lambda}^{Y}-T}$ 
where $S_{\lambda}^{Y}:[0,1]^{2} \rightarrow[0,1]$ is Yager's t-conorm family, that is $S_{\lambda}^{Y}(u, v)=\min \left(\left(u^{\lambda}+v^{\lambda}\right)^{1 / \lambda}, 1\right)$, obtained when $\lambda=1$. They provide an interesting means to define fuzzy equivalence relations when using linguistic hedges as proposed by De Cock and Kerre [11] (see an example in section 5.3 on the fairy-tale problem).

\section{Fuzzy partitions}

\subsection{Fuzzy partitions on $X \subset \mathfrak{R}$}

Proposition 10: Let $T$ be a strict t-norm with a strictly convex additive generator and a diagonal section $\phi$. Let $P=\left\{A_{i}\right\}_{i \in\{1, \ldots, n\}}$ be a $\phi$-partition on $X \subset \mathfrak{R}$. Let $D_{x}(i)=A_{i}(x)$ and $D_{y}(i)=A_{i}(y)$, for all $i \in\{1, \ldots, n\}$ and all $(x, y) \in X^{2}$. Then, $R_{T}\left(D_{x}, D_{y}\right)$ is, at least, a $T_{D}$-equivalence on $X$ where $T_{D}$ is the smallest t-norm.

Proof. Because $P$ is a $\phi$-partition on $X, R_{T}$ is reflexive on $X$. It is symmetric by definition. Because the additive generator is strictly convex, the relation is at least $T_{D}$-transitive on $X$ according to proposition 6 .

Proposition 11: Let $T$ be a strict t-norm with a convex additive generator and a diagonal section $\phi$. Let $I=\{1, \ldots, n\}$ and $P=\left\{A_{i}\right\}_{i \in I}$ be a $\phi$-partition on $X \subset \Re$. Let $J=\left\{J_{k}\right\}_{k \in K}$ be a partition on the set I. Let $S^{*}:[0,1]^{2} \rightarrow[0,1]$ be the t-conorm isomorphic to Lukasiewicz $t$-conorm defined by: $S^{*}(u, v)=\phi^{-1}\left(S_{L}(\phi(u), \phi(v))\right)$ with $S_{L}(u, v)=\min (u+v, 1)$, then $P^{\prime}=\left\{B_{k}\right\}_{k \in K}$, with $B_{k}(x)=S^{*}{ }_{i \in J_{k}}\left(A_{i}(x)\right)$, is a $\phi$-partition on $X \subset \Re$.

Proof. Let us write the condition for $P$ ' to be a $\phi$-partition on $X$ :

$$
\sum_{k \in K} \phi\left(B_{k}(x)\right)=\sum_{k \in K} \phi\left(S^{*}{ }_{i \in J_{k}}\left(A_{i}(x)\right)\right)=\sum_{k \in K} S_{L} i_{i \in J_{k}} \phi\left(A_{i}(x)\right)=\sum_{k \in K} \min \left(\sum_{i \in J_{k}} \phi\left(A_{i}(x)\right), 1\right) .
$$

Since $P$ is $\phi$-partition on $X$, we have $\sum_{i \in J_{k}} \phi\left(A_{i}(x)\right) \leq 1$ and, therefore, because $J$ is a partition on $I$, we have:

$$
\sum_{k \in K} \phi\left(B_{k}(x)\right)=\sum_{k \in K} \sum_{i \in J_{k}} \phi\left(A_{i}(x)\right)=1
$$


Remark 8: This proposition will be very useful to build fuzzy partition, on cartesian products, for rule-based agregation as described in section 5.5 .

Definition 5: An (L-R) fuzzy interval [15] (see figure 3) is defined by the following membership function:

$A(x)= \begin{cases}L\left(\frac{a-x}{\alpha}\right) & \text { if } x \in] a-\alpha, a[ \\ 1 & \text { if } x \in[a, b], \\ R\left(\frac{x-b}{\beta}\right) & \text { if } x \in] b, b+\beta[ \\ 0 & \text { otherwise }\end{cases}$

where $a, b \in \mathfrak{R}, a \leq b$ and $\alpha, \beta \in[0, \infty[$ and $L, R$ two non-increasing left-continuous functions from $] 0,1]$ to $[0,1[$ with $L(x)>0$ and $R(x)>0$ for all $x \in] 0,1[$.

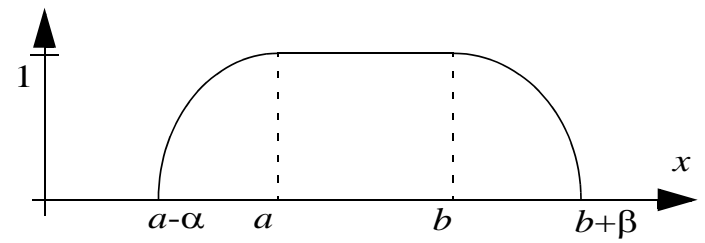

Fig. 3 A $(L-R)$ fuzzy interval.

Proposition 12: Let $T$ be a strict t-norm with a diagonal section $\phi . \quad$ Let $P=\left\{A_{i}\right\}_{i \in\{1, \ldots, n\}}=\left\{\left(a_{i}, b_{i}, \alpha_{i}, \beta_{i}\right)_{L R}\right\}_{i \in\{1, \ldots, n\}}$ be a family of $(L-R)$ fuzzy intervals defined on $\left[a_{1}, b_{n}\right] \subset \Re$, with $L(u)=R(u)=\phi^{-1}(1-u)$ for all $\left.\left.u \in\right] 0,1\right]$, and such that:

$b_{i}=a_{i+1}-\alpha_{i+1}$,

$b_{i}+\beta_{i}=a_{i+1}$.

Then, for all $i \in\{1, \ldots, n-1\}$ and all $x \in] b_{i}, a_{i+1}\left[\right.$, we have $L\left(\frac{a_{i+1}-x}{\alpha_{i+1}}\right)=\phi^{-1}\left(1-\phi\left(R\left(\frac{x-b_{i}}{\beta_{i}}\right)\right)\right)$.

Proof. For all $i \in\{1, \ldots, n\}$, we have $\alpha_{i+1}=\beta_{i}$. Now, for all $i \in\{1, \ldots, n-1\}$ and all $\left.x \in\right] b_{i}, a_{i+1}[$ we have:

$$
\begin{aligned}
& \phi^{-1}\left(1-\phi\left(R\left(\frac{x-b_{i}}{\beta_{i}}\right)\right)\right)=\phi^{-1}\left(1-\phi\left(\phi^{-1}\left(1-\frac{x-b_{i}}{\beta_{i}}\right)\right)\right)=\phi^{-1}\left(\frac{x-b_{i}}{\beta_{i}}\right)=\phi^{-1}\left(1-\frac{\alpha_{i+1}+b_{i}-x}{\alpha_{i+1}}\right) \\
& =\phi^{-1}\left(1-\frac{a_{i+1}-x}{\alpha_{i+1}}\right)=L\left(\frac{a_{i+1}-x}{\alpha_{i+1}}\right) .
\end{aligned}
$$


Corollary 2: $P$ is a $\phi$-partition on $\left[a_{1}, b_{n}\right] \subset \Re$.

Proof. For all $i \in\{1, \ldots, n\}$ and for all $x \in\left[a_{i}, b_{i}\right], \sum_{j=1} \phi\left(A_{j}(x)\right)=1$. Now, for all $i \in\{1, \ldots, n-1\}$ and all $x \in] b_{i}, a_{i+1}[$ we have:

$\sum_{j=1}^{n} \phi(j(x))=\phi\left(R\left(\frac{x-b_{i}}{\beta_{i}}\right)\right)+\phi\left(L\left(\frac{a_{i+1}-x}{\alpha_{i+1}}\right)\right)=\phi\left(R\left(\frac{x-b_{i}}{\beta_{i}}\right)\right)+\phi\left(\phi^{-1}\left(1-\phi\left(R\left(\frac{x-b_{i}}{\beta_{i}}\right)\right)\right)\right)=1$.

Remark 9: This generic method to build the fuzzy partitions associated with $R_{T}$ relations is a straightforward consequence of the definition of a $\phi$-partition from an algebraic (or strict) fuzzy partition [8]. For the same reason, it can be generalized by using a mapping $\phi^{\prime^{-1}}(u)=\phi^{-1}(h(u))$ where $h:[0,1] \rightarrow[0,1]$, is a strictly increasing function with $h(0)=0$ and $h(1)=1$, such that:

$h(u)+h(1-u)=1$

4.2 Fuzzy partition on $X_{1} \times X_{2} \subset \mathfrak{R}^{2}$

Proposition 13: Let $T$ be a strict t-norm with a strictly convex additive generator and a diagonal section $\phi$. Let $I=\{1, \ldots, n\}$ and $J=\{1, \ldots, m\}$. Let $P_{1}=\left\{A_{i}\right\}_{i \in I}$ and $P_{2}=\left\{B_{j}\right\}_{j \in J}$ be two $\phi$-partitions respectively on $X_{1}$ and $X_{2}$. Let $T^{*}:[0,1]^{2} \rightarrow[0,1]$ be the strict t-norm defined by $T^{*}(u, v)=\phi^{-1}(\phi(u) \cdot \phi(v))$. Then, $P=\left\{C_{(i, j)}\right\}_{(i, j) \in I \times J}$ such that for all $\left(x_{1}, x_{2}\right) \in X_{1} \times X_{2}$, $C_{(i, j)}\left(x_{1}, x_{2}\right)=T^{*}\left(A_{i}\left(x_{1}\right), B_{j}\left(x_{2}\right)\right)$, is a $\phi$-partition on $X_{1} \times X_{2}$.

Proof. According to proposition 10, for all $\left(x_{1}, y_{1}\right) \in X_{1}^{2} \subset \mathfrak{R}^{2}$ and $\left(x_{2}, y_{2}\right) \in X_{2}^{2} \subset \mathfrak{R}^{2}, R_{T}^{1}\left(D_{x_{1}}, D_{y_{1}}\right)$ and $R_{T}^{2}\left(D_{x_{2}}, D_{y_{2}}\right)$ are, at least, $T_{D}$-equivalences. Now, from proposition 9 we know that the relation defined by: 
$R_{T}\left(\left(D_{x_{1}}, D_{x_{2}}\right),\left(D_{y_{1}}, D_{y_{2}}\right)\right)=\sum_{i=1 j=1}^{n} \sum_{j}^{m} T\left(T^{*}\left(D_{x_{1}}(i), D_{x_{2}}(j)\right), T^{*}\left(D_{y_{1}}(i), D_{y_{2}}(j)\right)\right)$,

is a fuzzy equivalence relation on $X_{1} \times X_{2}$. The reflexivity condition leads to:

$R_{T}\left(\left(D_{x_{1}}, D_{x_{2}}\right),\left(D_{x_{1}}, D_{x_{2}}\right)\right)=1=\sum_{i=1 j=1}^{n} \sum_{1 j}^{m} \phi\left(T^{*}\left(D_{x_{1}}(i), D_{x_{2}}(j)\right)\right)=\sum_{(i, j) \in I \times J} \phi\left(C_{(i, j)}\left(x_{1}, x_{2}\right)\right)$.

Proposition 14: Let $T$ be a strict t-norm with a strictly convex additive generator and a diagonal section $\phi$. Let $I=\{1, \ldots, n\}$ and $J=\{1, \ldots, m\}$. Let $P_{1}=\left\{A_{i}\right\}_{i \in I}$ and $P_{2}=\left\{B_{j}\right\}_{j \in J}$ be two $\phi$-partitions respectively on $X_{1}$ and $X_{2}$. Then, $\forall i \in I, \forall(j, k) \in J^{2}$, we have for all $\left(x_{1}, x_{2}\right) \in X_{1} \times X_{2}$ :

$T^{*}\left(A_{i}\left(x_{1}\right), S^{*}\left(B_{j}\left(x_{2}\right), B_{k}\left(x_{2}\right)\right)\right)=S^{*}\left(T^{*}\left(A_{i}\left(x_{1}\right), B_{j}\left(x_{2}\right)\right), T^{*}\left(A_{i}\left(x_{1}\right), B_{k}\left(x_{2}\right)\right)\right)$.

Proof. For the sake of simplicity, let us denote $u=A_{i}\left(x_{1}\right), v=B_{j}\left(x_{2}\right), w=B_{k}\left(x_{2}\right)$. Replacing $T^{*}$ and $S^{*}$ by their respective definitions on the left hand side part leads to:

$T^{*}\left(u, S^{*}(v, w)\right)=\phi^{-1}\left(\phi(u) \cdot \phi\left(S^{*}(v, w)\right)\right)=\phi^{-1}\left(\phi(u) \cdot S_{L}(\phi(v), \phi(w))\right)$.

Similarly, replacing $S^{*}$ and $T^{*}$ on the right hand side part gives:

$S^{*}\left(T^{*}(u, v), T^{*}(u, w)\right)=\phi^{-1}\left(S_{L}(\phi(u) \cdot \phi(v), \phi(u) \cdot \phi(w))\right)$.

Because $P_{2}$ is a $\phi$-partition, we have $\phi(v)+\phi(w) \leq 1$ and, thus:

$S^{*}\left(T^{*}(u, v), T^{*}(u, w)\right)=\phi^{-1}\left(\phi(u) \cdot S_{L}(\phi(v), \phi(w))\right)$

Remark 10: It should be noted that equation (45) is not the conventional distributivity between $T^{*}$ and $S^{*}$ which is known to hold true only if $T^{*}=T_{M}$. Indeed, $B_{j}\left(x_{2}\right)$ and $B_{k}\left(x_{2}\right)$ are not independant since they belong to the same $\phi$-partition. However, it will be very interesting in applications because it allows to define a «linguistic distributivity» between connectives, which makes it possible to aggregate linguistic terms indifferently on the cartesian product $X=X_{1} \times X_{2}$ or on the sets $X_{1}$ or $X_{2}$, as shown in the next sections. 


\subsection{Linguistic labeling and connectives}

A few years after his seminal paper on fuzzy set theory, Zadeh introduced the concept of fuzzy meaning [28]. It can be represented by a mapping $M: L \rightarrow F(X)$, where $F(X)$ is the set of fuzzy subsets of $X$, given a relation between terms and numbers. The grade of membership to which $\mathrm{x}$ belongs to the meaning of the term $l$ will be denoted $M_{l}(x)$.

In [28], Zadeh introduced also the concept of descriptor set that are extensively used in fuzzy sensors [1][21]. It provides a simple means of representing measurement results by a fuzzy subset of linguistic terms. The conversion from numerical to linguistic representation is called fuzzy linguistic (or symbolic) description or, more simply, fuzzy description and is defined by a mapping $D: X \rightarrow F(L)$, where $F(L)$ is the set of fuzzy subsets of $L$, given the same relation between terms and numbers as the one used for the fuzzy meaning. The grade of membership to which $l$ belongs to the description of the number $x$ will be denoted $D_{x}(l)$. Fuzzy description is very close to the representation defined in the scale formalism [17] and allows the introduction of graduality in the conversion of physical states into fuzzy subsets of terms.

Example 4: Let us assume that a very simple sensor returns the size of a human being. Let the linguistic set be $L=\{$ Small, Medium, Tall $\}$ and the measurement set $X=\{1.4,1.5,1.6,1.7,1.8\}$ where the sizes are given in meters. A possible relation linking the size attributes to the measurements is given in table .

\begin{tabular}{|c|c|c|c|}
\hline & Small & Medium & Tall \\
\hline 1.4 & 1 & 0 & 0 \\
\hline 1.5 & 0.7 & 0.4 & 0 \\
\hline 1.6 & 0.3 & 0.8 & 0 \\
\hline 1.7 & 0.1 & 1 & 0.3 \\
\hline 1.8 & 0 & 0.8 & 0.7 \\
\hline
\end{tabular}

Table 4: Fuzzy relation between the linguistic set $L$ and the measurement set $X$.

Then, using the conventional additive notation for discrete fuzzy subsets, we have:

$M_{\text {Small }}=1 / 1.4+0.7 / 1.5+0.3 / 1.6+0.1 / 1.7+0 / 1.8$ and $D_{1.7}=0.1 /$ Small $+1 /$ Medium $+0.3 /$ Tall .

Remark 11: The fuzzy meaning and the fuzzy description are two different projections of the same relation. Therefore, for all $l \in L$ and all $x \in X$, we have $M_{l}(x)=D_{x}(l)$. 
Remark 12: The fuzzy description of number $x$ is an element of $F(L)$ and, therefore a vector in $[0,1]^{n}$ if $\operatorname{card}(L)=n$. Thus, the fuzzy description provides a simple means to build fuzzy equivalence relations on set of numbers. The partitions associated with these equivalence relations are given by the fuzzy meaning of the terms. Indeed, given a set $P=\left\{A_{i}\right\}_{i \in I}$ of membership functions with $I=\{1, \ldots, n\}$, we can always define a bijection $f: I \rightarrow L$ such that, for all $i \in I, M_{l}=A_{f(i)}$. The use of a set linguistic terms $l \in L$ and their fuzzy meaning $M_{l}$ is nothing more than a re-labeling of the membership function $A_{i}$ which will be more convenient to develop applications.

Definition 6: Let T be a strict t-norm with a strictly convex additive generator and a diagonal section $\phi$. Let $L_{1}$ and $L_{2}$ be two sets of linguistic terms whose fuzzy meanings are defined respectively on $X_{1}$ and $X_{2}$ and denoted $M_{l}$, for all $l_{1} \in L_{1}$ and $l_{2} \in L_{2}$. Let $T^{*}:[0,1]^{2} \rightarrow[0,1]$ be the strict t-norm defined by $T^{*}(u, v)=\phi^{-1}(\phi(u) \cdot \phi(v))$. For all $\left(l_{1}, l_{2}\right) \in L_{1} \times L_{2}$, we will say that $T^{*}$ defines the meaning of a new term « $l_{1}$ and $l_{2} »$ on $L_{1} \times L_{2}$ as follows: $\forall\left(x_{1}, x_{2}\right) \in X_{1} \times X_{2}, M_{l_{1} \text { and } l_{2}}\left(x_{1}, x_{2}\right)=T^{*}\left(M_{l_{1}}\left(x_{1}\right), M_{l_{2}}\left(x_{2}\right)\right)$.

Definition 7: Let $T$ be a strict t-norm with a strictly convex additive generator and a diagonal section $\phi$. Let $L$ be a set of linguistic terms whose fuzzy meanings are defined on $X$ and denoted $M_{l}$, for all $l \in L$. Let $S^{*}:[0,1]^{2} \rightarrow[0,1]$ be the t-conorm, isomorphic to Lukasiewicz t-conorm, defined by $S^{*}(u, v)=\phi^{-1}\left(S_{L}(\phi(u), \phi(v))\right)$, where $S_{L}(u, v)=\min (u+v, 1)$. For all $\left(l_{1}, l_{2}\right) \in L^{2}$, we will say that $S^{*}$ defines the meaning of a new term « $l_{1}$ or $l_{2} »$ on $L$ as follows:

$\forall x \in X, M_{l_{1} \text { or } l_{2}}(x)=S^{*}\left(M_{l_{1}}(x), M_{l_{2}}(x)\right)$.

Definition 8: Let $T$ be a strict t-norm with a strictly convex additive generator and a diagonal section $\phi$. Let $L$ be a set of linguistic terms. Let $\left\{M_{l}\right\}_{l \in L}$ be a $\phi$-partition on $X$, with $M_{l}$ the fuzzy meanings of the term l, such that, for all all $l_{1} \in L$, there exists $l_{2} \in L$ and $\forall x \in X, \phi\left(M_{l_{1}}(x)\right)+\phi\left(M_{l_{2}}(x)\right)=1$. Let $N^{*}:[0,1] \rightarrow[0,1]$ defined by $N^{*}(u)=\phi^{-1}(1-\phi(u))$. For all $l \in L$, we will say that $N^{*}$ defines the meaning of a new term «not $l »$ on $L$ as follows:

$\forall x \in X, M_{\text {not } l}(x)=N^{*}\left(M_{l}(x)\right)$. 
Remark 13: The negation $N^{*}$ can be used with $\phi$-partitions defined by means of $(L-R)$ intervals as shown in section 4.1. Let us note also that the triplet $\left(S^{*}, T^{*}, N^{*}\right)$ obtained by means of the bijection $\phi$ satisfies the functional equation of Alsina [7], that is $S^{*}\left(T^{*}(u, v), T^{*}(u, N(v))=u\right.$, for all $u=A_{i}$ and $v=B_{j}$, with $\left\{A_{i}\right\}_{i \in I}$ and $\left\{B_{j}\right\}_{j \in J}$ two $\phi$-partitions respectively on $X_{1}$ and $X_{2}$. The proof is obvious using proposition 14 .

\section{Examples}

\subsection{Fuzzy partitions on $\mathfrak{R}$}

Figure 4 represents three fuzzy partitions and their associated fuzzy equivalence relations generated according to the principle given in section 4.1. They are respectively obtained with the t-norm $T_{M}, T_{500}^{F}$ with $h(x)=x$ and $T_{M}$ with $h(x)=\sin ^{2}\left(\frac{\pi}{2} x\right)$
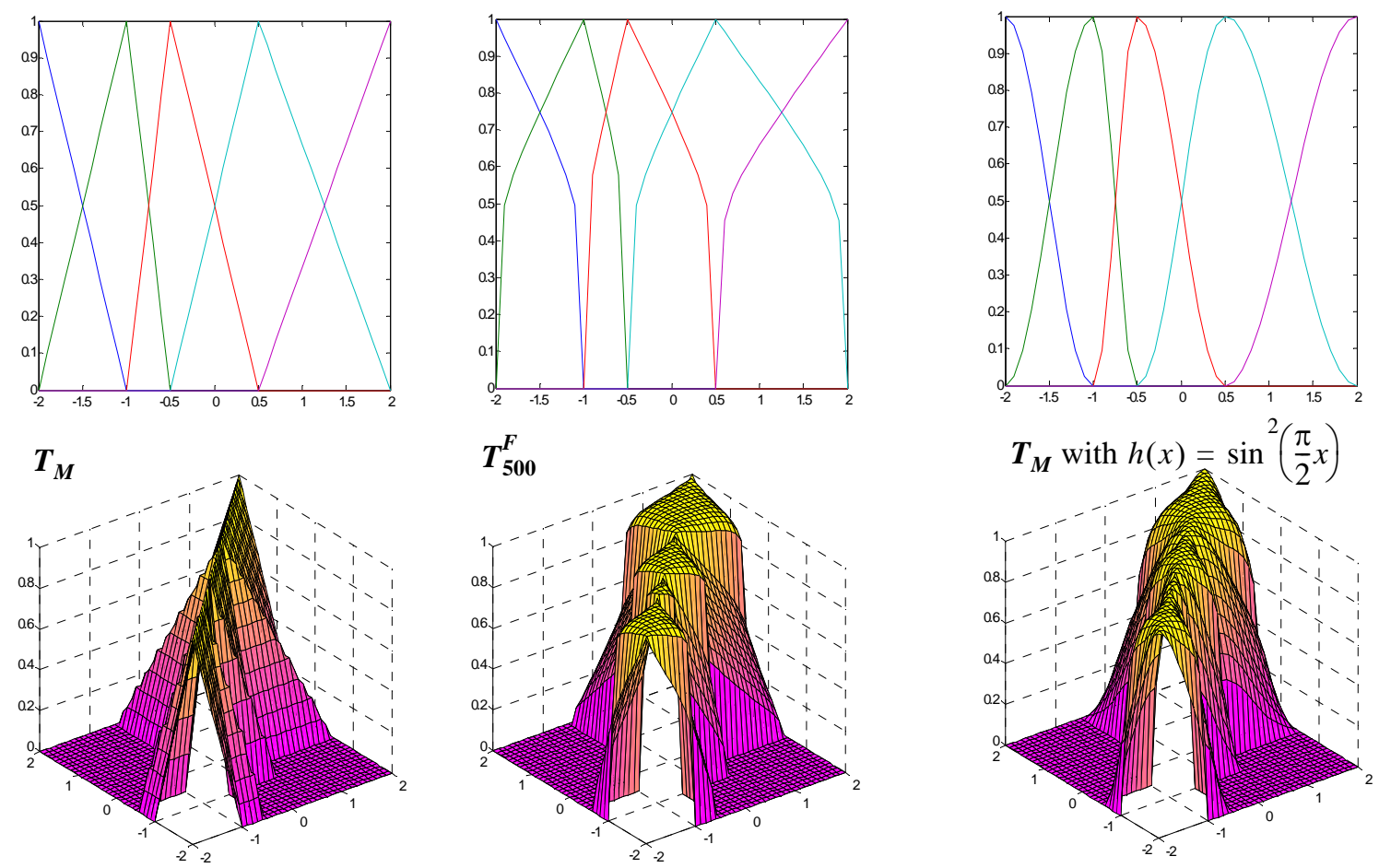

Fig. 4 Examples of fuzzy partitions and their associated equivalence relations.

\subsection{Comparing colors}

Let us analyze a more complex example where colored objects have to be compared. It will be assumed that the color information comes from a sensor based on three photo-detectors recreating the effects of the red, green, blue cones of the human eyes. When the sensor information is normalized, the color space is simply defined as the unit cube $(R, G, B)$. In order to allow a simple description of colors, the luminosity will be separated from the 
chrominance information by a non linear mapping as shown in figure 5.

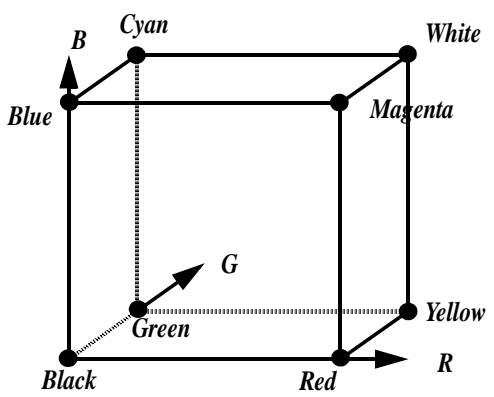

$$
\begin{gathered}
{\left[\begin{array}{l}
x_{1} \\
x_{2}
\end{array}\right]=\left[\begin{array}{rrr}
1 & -\frac{1}{2} & -\frac{1}{2} \\
0 & -\frac{\sqrt{3}}{2} & \frac{\sqrt{3}}{2}
\end{array}\right]\left[\begin{array}{l}
\frac{r}{\mathrm{Y}^{\prime}} \\
\frac{v}{\mathrm{Y}^{\prime}} \\
\frac{b}{\mathrm{Y}^{\prime}}
\end{array}\right]} \\
\mathrm{Y}^{\prime}=\max (r, v, b)
\end{gathered}
$$

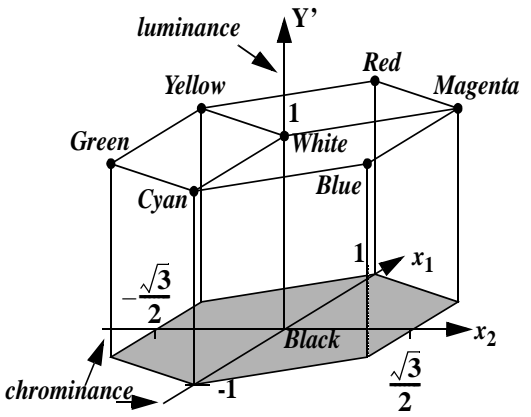

Fig. 5 From the $(R, G, B)$ cube to the Chrominance-Luminance representation

Now let us assume that the Delaunay triangulation of the chrominance plane is used to perform a multi-linear interpolation that defines the 2D-fuzzy meanings of the linguistic terms, as shown in figure 6 for the two terms Red and Grey [1]. The origin of the chrominance plane is labelled with the term Grey and the luminance information should be used to distinguish grey levels from Black to White.
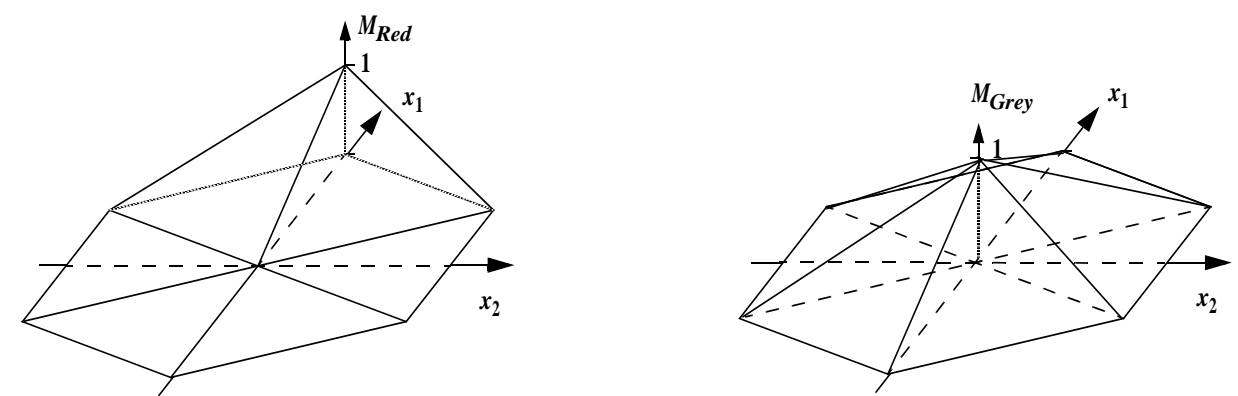

Fig. 6 The 2D-Fuzzy meaning of the terms Red and Grey.

This representation verifies the property:

$\forall\left(x_{1}, x_{2}\right) \in X \subset \mathfrak{R}^{2}, \sum_{l \in L} D_{\left(x_{1}, x_{2}\right)}(l)=\sum_{l \in L} M_{l}\left(x_{1}, x_{2}\right)=1$,

where $L=\{$ Red, Yellow, Grey, Magenta, Cyan, Blue, Green $\}$.

Now, let us assume that $O=\{A, B, C, D, E, F\}$ is a set of six objects to be analyzed by a fuzzy color sensor. Let $\left(x_{1}^{o}, x_{2}^{o}\right)$ be the chrominance information associated with an object $o \in O$, that is $\operatorname{Color}(o)=\left(x_{1}^{o}, x_{2}^{o}\right)$. The fuzzy sensor provides the fuzzy description of the chrominance information associated with each object, thus we have:

$\forall o \in O, D_{\text {Color }(o)}=D_{\left(x_{1}^{o}, x_{2}^{o}\right)}$ 
The position of each object in the chrominance plane is represented in figure 7.

\begin{tabular}{ccc}
\hline & $x_{1}$ & $x_{2}$ \\
\hline$A$ & 0.1 & 0.8 \\
$B$ & 0.4 & 0.6 \\
$C$ & 0.6 & -0.35 \\
$D$ & 0.5 & -0.8 \\
$E$ & -0.3 & 0.7 \\
$F$ & 0 & 0 \\
\hline
\end{tabular}

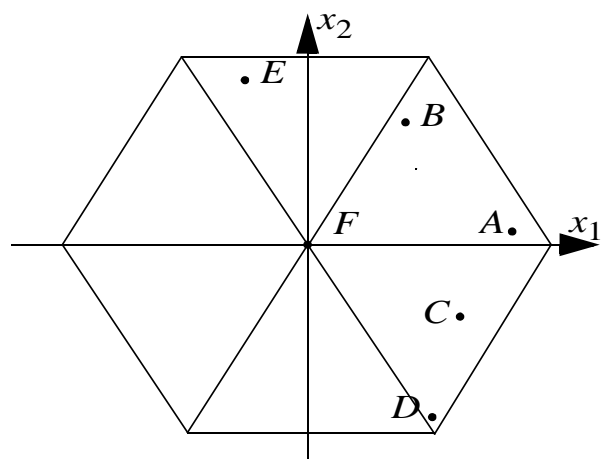

Fig. 7 Objects in the chrominance plane

The fuzzy description of the pair $\left(x_{1}, x_{2}\right)$ associated with each object is given in table 6 . For example, we have:

$D_{\text {Color }(A)}=D_{(0.1,0.8)}=0.1423 /$ Grey $+0.7423 /$ Red $+0.1154 /$ Magenta

\begin{tabular}{cccccccc}
\hline & Grey & Red & Magenta & Blue & Cyan & Green & Yellow \\
\hline A & 0.1423 & 0.7423 & 0.1154 & 0 & 0 & 0 & 0 \\
$B$ & 0.2536 & 0.0536 & 0.6928 & 0 & 0 & 0 & 0 \\
$C$ & 0.1979 & 0.3979 & 0 & 0 & 0 & 0 & 0.4042 \\
$D$ & 0.0381 & 0.0381 & 0 & 0 & 0 & 0 & 0.9238 \\
$E$ & 0.1916 & 0 & 0.1042 & 0.7042 & 0 & 0 & 0 \\
$F$ & 1 & 0 & 0 & 0 & 0 & 0 & 0 \\
\hline
\end{tabular}

Table 6: Fuzzy descriptions of the colors of the six objects

Thanks to the property given by Eq. (49), a $T_{L}$-equivalence can be obtained from the following $R_{T_{M}}$ fuzzy relation:

$$
\begin{aligned}
\forall\left(o_{1}, o_{2}\right) \in O^{2}, \quad R_{T_{M}}\left(D_{\text {Color }\left(o_{1}\right)}, D_{\text {Color }\left(o_{2}\right)}\right) & =\sum_{l \in L} \min \left(D_{\operatorname{Color}\left(o_{1}\right)}(l), D_{\operatorname{Color}\left(o_{2}\right)}(l)\right) \\
& =\sum_{l \in L} \min \left(D_{\left(x_{1}^{o_{1}}, x_{2}{ }^{1}\right)}(l), D_{\left(x_{1}^{o_{2}}, x_{2}\right)^{2}}(l)\right)
\end{aligned}
$$

When there is no ambiguity, it can be expedient to abbreviate Attribute(o) to $o$, relying on the context for the determination of whether $o$ stands for an object or for its attribute. Thus, for the sake of readibility, the results given in table 7 are labelled with the objects instead of their color (e.g. $A$ is used in place of Color(A)). 


\begin{tabular}{ccccccc}
\hline$R_{T_{M}}$ & $A$ & $B$ & $C$ & $D$ & $E$ & $F$ \\
\hline$A$ & 1.0000 & 0.3113 & 0.5402 & 0.0762 & 0.2465 & 0.1423 \\
$B$ & 0.3113 & 1.0000 & 0.2515 & 0.0762 & 0.2958 & 0.2536 \\
$C$ & 0.5402 & 0.2515 & 1.0000 & 0.4804 & 0.1916 & 0.1979 \\
$D$ & 0.0762 & 0.0762 & 0.4804 & 1.0000 & 0.0381 & 0.0381 \\
$E$ & 0.2465 & 0.2958 & 0.1916 & 0.0381 & 1.0000 & 0.1916 \\
$F$ & 0.1423 & 0.2536 & 0.1979 & 0.0381 & 0.1916 & 1.0000 \\
\hline
\end{tabular}

Table 7: $R_{T_{M}}$ fuzzy relation for the color example.

This $R_{T_{M}}$ fuzzy relation is a $T_{L}$-equality, therefore $d=t_{L}$ o $R_{T_{M}}$ is a metric on the set $X$, where $t_{L}$ is the additive generator of the t-norm $T_{L}$, that is $t_{L}(u)=1-u$ (see [18] for example). Thus, it makes it possible to compare objects in terms of distance in the color space:

$\forall\left(o_{1}, o_{2}\right) \in O^{2}, d\left(\operatorname{Color}\left(o_{1}\right), \operatorname{Color}\left(o_{2}\right)\right)=1-R_{T_{M}}\left(D_{\operatorname{Color}\left(o_{1}\right)}, D_{\operatorname{Color}\left(o_{2}\right)}\right)$

For example, the color of $B$ is closer to the color of $A$ than that of $C$ because we have $d(\operatorname{Color}(B), \operatorname{Color}(A))=0.6887$ and $d(\operatorname{Color}(B), \operatorname{Color}(C))=0.7485$

\subsection{Comparing beauty}

In [12], De Cock and Kerre have proposed an interesting example where fairy-tale characters, belonging to the set $O=\{$ Snowwhite, Witch, Wolf, Dwarf, Prince, Little-Red-Riding-Hood $\}$, have their beauty compared (see also comments on this paper [4], [5], [13], [20], [19]). Available information is given by the following table:

\begin{tabular}{lccc}
\hline & Beautiful & Average & Ugly \\
\hline Snowwhite & 1.00 & 0.00 & 0.00 \\
Witch & 0.00 & 0.30 & 0.70 \\
Wolf & 0.00 & 0.00 & 1.00 \\
Dwarf & 0.10 & 0.70 & 0.20 \\
Prince & 0.80 & 0.20 & 0.00 \\
Red-Riding-Hood & 0.50 & 0.50 & 0.00 \\
\hline
\end{tabular}

Table 8: the fairy-tale characters.

Rather than interpreting this table as «the fuzzy sets beautiful, average and ugly in O», as suggested in [12], we will consider it as the fuzzy linguistic descriptions of the characters' beauty (Let us note that in [12] the set of the fairy-tale characters is denoted $X$ which has another meaning in this paper). More formally, this means that the beauty of one character in $O$ is a fuzzy subset defined on the set $L=\{$ Beautiful, Average, Ugly $\}$. In other words, 
we have a horizontal reading of the table instead of a vertical one. For example, let us write the beauty of the Dwarf as Beauty(Dwarf). It is assumed that Beauty(Dwarf) is an unknown piece of information but whose fuzzy description is known and given by:

$D_{\text {Beauty (Dwarf) }}=0.10 /$ Beautiful $+0.70 /$ Average $+0.20 /$ Ugly

Since the linguistic description of beauty is given by a human being, it will be supposed that he/she uses fuzzy meanings of Beautiful, Average and Ugly resulting from a non-explicit aggregation of several criteria of beauty and it will be assumed that he/she provides coherent information.

As can be observed, the sum of the grades of memberships for each line of table 8 is equal to one. Therefore, a $T_{L}$-equivalence is obtained from the following $R_{T_{M}}$ fuzzy relation:

$\forall\left(o_{1}, o_{2}\right) \in O^{2}, \quad R_{T_{M}}\left(D_{\text {Beauty }\left(o_{1}\right)}, D_{\text {Beauty }\left(o_{2}\right)}\right)=\sum_{l \in L} \min \left(D_{\text {Beauty }\left(o_{1}\right)}(l), D_{\text {Beauty }\left(o_{2}\right)}(l)\right)$

The resulting table for the fairy-tale characters is given in Table 9 which is exactly the same as the one given in [12].

\begin{tabular}{lcccccc}
\hline$R_{T_{M}}$ & Snowwhite & Witch & Wolf & Dwarf & $\begin{array}{c}\text { Prince } \\
\text { Red-Riding- } \\
\text { Hood }\end{array}$ \\
\hline Snowwhite & 1.00 & 0.00 & 0.00 & 0.10 & 0.80 & 0.50 \\
Witch & 0.00 & 1.00 & 0.70 & 0.50 & 0.20 & 0.30 \\
Wolf & 0.00 & 0.70 & 1.00 & 0.20 & 0.00 & 0.00 \\
Dwarf & 0.10 & 0.50 & 0.20 & 1.00 & 0.30 & 0.60 \\
Prince & 0.80 & 0.20 & 0.00 & 0.30 & 1.00 & 0.70 \\
Red-Riding- & 0.50 & 0.30 & 0.00 & 0.60 & 0.70 & 1.00 \\
Hood & & & & & & \\
\hline
\end{tabular}

Table 9: The fairy-tale $R_{T_{M}}$ fuzzy relation.

As for the color example, a distance can be associated with this equivalence relation in order to compare the beauty of the fairy-tale characters:

$\forall\left(o_{1}, o_{2}\right) \in O^{2}, d\left(\operatorname{Beauty}\left(o_{1}\right), \operatorname{Beauty}\left(o_{2}\right)\right)=1-R_{T_{M}}\left(D_{\text {Beauty }\left(o_{1}\right)}, D_{\text {Beauty }\left(o_{2}\right)}\right)$.

As mentioned in section 3.5, $R_{T}$ fuzzy relations are a particular case of the parametrized family $R_{S_{\lambda}^{Y}-T}$ where $S_{\lambda}^{Y}$ is Yager's t-conorm family. It provides an interesting feature to deal with linguistic hedges based on the powering of the membership functions [11]: 
$M_{h(l)}(x)=P_{\alpha}\left(M_{l}(x)\right)=\left(M_{l}(x)\right)^{\alpha}$, where $h$ is a linguistic hedge [29]

Indeed, under the conditions for proposition 2, we have:

$$
\begin{aligned}
& \forall \lambda>0, R_{S_{\lambda}^{Y}-T}\left(D_{x}, D_{y}\right)=\min \left(\left(\sum_{l \in L} T\left(\left(M_{h(l)}(x)\right)^{\lambda},\left(M_{h(l)}(y)\right)^{\lambda}\right)\right)^{1 / \lambda}, 1\right) \\
& =\min \left(\left(\sum_{l \in L} T\left(P_{1 / \lambda}\left(M_{l}(x)\right)^{\lambda}, P_{1 / \lambda}\left(M_{l}(y)\right)\right)^{\lambda}\right)^{1 / \lambda}, 1\right) \\
& =P_{1 / \lambda}\left(R_{T}(x, y)\right)
\end{aligned}
$$

The $R_{S_{\lambda}^{Y}-T}$ fuzzy relation built using the membership functions modified by powering hedges is the $R_{T}$ fuzzy relation raised to the power of the hedge as represented in figure 8.

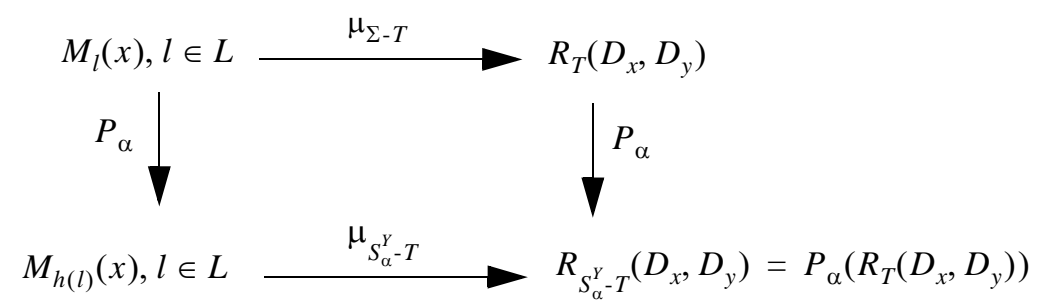

Fig. 8 Links between powering hedges and $R_{S_{\alpha}^{Y}-T}$ fuzzy relations.

Table 10 illustrates the equivalence relation generated when using the membership functions obtained with the linguistic hedges very defined as $P_{2}$. It means that the fuzzy descriptions of the characters' beauty are defined on

\begin{tabular}{|c|c|c|c|c|c|c|}
\hline$R_{S_{2}^{Y}-T_{M}}$ & Snowwhite & Witch & Wolf & Dwarf & Prince & $\begin{array}{c}\text { Red-Riding- } \\
\text { Hood }\end{array}$ \\
\hline Snowwhite & 1.00 & 0.00 & 0.00 & 0.01 & 0.64 & 0.25 \\
\hline Witch & 0.00 & 1.00 & 0.49 & 0.25 & 0.04 & 0.09 \\
\hline Wolf & 0.00 & 0.49 & 1.00 & 0.04 & 0.00 & 0.00 \\
\hline Dwarf & 0.01 & 0.25 & 0.04 & 1.00 & 0.09 & 0.36 \\
\hline Prince & 0.64 & 0.04 & 0.00 & 0.09 & 1.00 & 0.49 \\
\hline $\begin{array}{l}\text { Red-Riding- } \\
\text { Hood }\end{array}$ & 0.25 & 0.09 & 0.00 & 0.36 & 0.49 & 1.00 \\
\hline
\end{tabular}
the set $L^{\prime}=\{$ Very_Beautiful, Very_Average, Very_Ugly $\}$ as for example:

$\mathrm{D}_{\text {Beauty(Dwarf) }}=0.01 /$ Very_Beautiful $+0.49 /$ Very_Average $+0.04 /$ Very_Ugly .

Table 10: The fairy-tale fuzzy equivalence relation using the linguistic hedge very.

\subsection{Fairy-tale characters with colored clothes}

In order to illustrate a $R_{T}$ fuzzy equivalence relation on a cartesian product, we will compare fairy tale 
characters with regard to their beauty and the color of their clothes. The objects whose color were described in section 5.2 are considered as fairy-tale characters' clothes according to table 11.

\begin{tabular}{cc}
\hline Clothes & Object \\
\hline Snowwhite & $D$ \\
Witch & $F$ \\
Wolf & $C$ \\
Dwarf & $B$ \\
Prince & $E$ \\
Red-riding-Hood & $A$
\end{tabular}

Table 11: Fairy-tale characters' clothes.

Let $O=\{$ Snowwhite, Witch, Wolf, Dwarf, Prince, Little-red-riding-Hood $\}, L_{1}=\{$ Beautiful, Average, Ugly $\}$ and $L_{2}=\{$ Red, Yellow, Grey, Magenta, Cyan, Blue, Green $\}$, the $R_{T_{M}}$ fuzzy relation is given by:

$\forall\left(o_{1}, o_{2}\right) \in O^{2}, \quad R_{T_{M}}\left(\left(D_{\text {Beauty }\left(o_{1}\right)}, D_{\text {Color }\left(\operatorname{Clothes}\left(o_{1}\right)\right)}\right),\left(D_{\text {Beauty }\left(o_{2}\right)}, D_{\text {Color }\left(\operatorname{Clothes}\left(o_{2}\right)\right)}\right)\right)=$

$\sum_{l_{1} \in L_{1} l_{2} \in L_{2}} \sum_{\min }\left(T^{*}\left(D_{\text {Beauty }\left(o_{1}\right)}\left(l_{1}\right), D_{\text {Color }\left(\operatorname{Clothes}\left(o_{1}\right)\right)}\left(l_{2}\right)\right), T^{*}\left(D_{\text {Beauty }\left(o_{2}\right)}\left(l_{1}\right), D_{\operatorname{Color}\left(\operatorname{Clothes}\left(o_{2}\right)\right)}\left(l_{2}\right)\right)\right)$.

When using the t-norm $T=T_{M}$, we have $\phi(u)=u$ and, therefore, $T^{*}=T_{P}$, which leads to the following table. Let us note that, for the sake of simplicity, the table is labeled with the character's name instead of the pair (Beauty (o), Color(Clothes $(o)$ ) where $o$ would be the character's name.

\begin{tabular}{lllllll}
\hline$R_{T_{M}}$ & Snowwhite & Witch & Wolf & Dwarf & Prince & $\begin{array}{l}\text { Red-riding- } \\
\text { Hood }\end{array}$ \\
\hline Snowwhite & 1.0000 & 0.0000 & 0.0000 & 0.0307 & 0.0381 & 0.0762 \\
Witch & 0.0000 & 1.0000 & 0.1979 & 0.2282 & 0.0383 & 0.0712 \\
Wolf & 0.0000 & 0.1979 & 1.0000 & 0.0614 & 0.0000 & 0.0000 \\
Dwarf & 0.0307 & 0.2282 & 0.0614 & 1.0000 & 0.1538 & 0.2548 \\
Prince & 0.0381 & 0.0383 & 0.0000 & 0.1538 & 1.0000 & 0.1880 \\
Red-riding- & 0.0762 & 0.0712 & 0.0000 & 0.2548 & 0.1880 & 1.0000 \\
Hood & & & & & & \\
\hline
\end{tabular}

Table 12: The fairy-tale $R_{T_{M}}$ fuzzy equivalence relation on the cartesian product.

As can be noticed, the Prince's beauty was quite similar to Snowwhite's in table 9 but their respective clothes have different colors leading to a low similarity in the cartesian product. 


\subsection{Comparing comfort}

In this section, the aggregation of temperature and humidity to build comfort information [2] is proposed to illustrate fuzzy partitions on a cartesian product $X_{1} \times X_{2}$ where $X_{1}$ and $X_{2}$ are respectively associated with the temperature and the humidity. Let $L_{1}=\{$ Cold, Cool, Mild, Warm, Hot $\}, L_{2}=\{$ Very_Low, Low, Medium, High $\}$ and $L=\{$ Comfortable, Acceptable, Uncomfortable $\}$ be the sets of linguistic terms associated respectively with the temperature, the humidity and the comfort. As explained in section 4.2, the fuzzy meanings are obtained by the union of cartesian products of membership functions $X_{1}$ and $X_{2}$. It can be interpreted as a set of linguistic rules represented in figure 9. The black cell corresponds to the term Comfortable, while the grey and white ones are respectively associated with Acceptable and Uncomfortable.

\begin{tabular}{|c||c|c|c|c|}
\cline { 2 - 5 } \multicolumn{1}{c||}{} & Very_Low & Low & Medium & High \\
\hline \hline Hot & & & & \\
\hline Warm & & & & \\
\hline Mild & & & & \\
\hline Cool & & & & \\
\hline Cold & & & & \\
\hline
\end{tabular}

Fig. 9 Linguistic terms associated with comfort.

The definition of the terms Comfortable and Acceptable are given by:

Comfortable $=$ Mild and Medium.

Acceptable $=(\mathrm{Cool}$ and Low $)$ or (Mild and Low) or (Warm and Low) or (Cool and Medium) or (Warm and Medium).

Thanks to the «linguistic distributivity» of the connective and with respect to the linguistic connective or (see proposition 14), the latter definition can be rewritten as:

Acceptable $=((\mathrm{Cool}$ or Mild or Warm $)$ and Low $)$ or $((\mathrm{Cool}$ or Warm $)$ and Medium $)$.

The definition of the term Uncomfortable could be obtained similarly. However, it is simpler to use the negation connective and write:

Uncomfortable $=\operatorname{not}($ Comfortable or Acceptable $)$.

The fuzzy meanings of the linguistic terms on $X_{1}, X_{2}$ and $X_{1} \times X_{2}$ for $T=T_{P}$ are represented in figure 10 . Now, let $S_{1}, S_{2}, S_{3}, S_{4}$ be four comfort situations characterized by the following measurements: 


\begin{tabular}{ccc}
\hline Situation & Temperature & Humidity \\
\hline$S_{1}$ & $17{ }^{\circ} \mathrm{C}$ & $65 \%$ \\
$S_{2}$ & $28{ }^{\circ} \mathrm{C}$ & $30 \%$ \\
$S_{3}$ & $22^{\circ} \mathrm{C}$ & $45 \%$ \\
$S_{4}$ & $20^{\circ} \mathrm{C}$ & $55 \%$ \\
\hline
\end{tabular}

Table 13: Comfort situations.

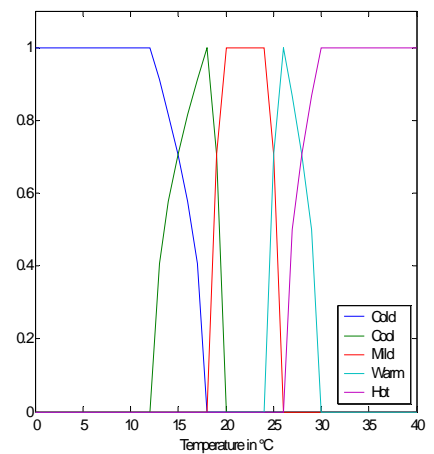

$$
T=T_{P}
$$

Comfortable
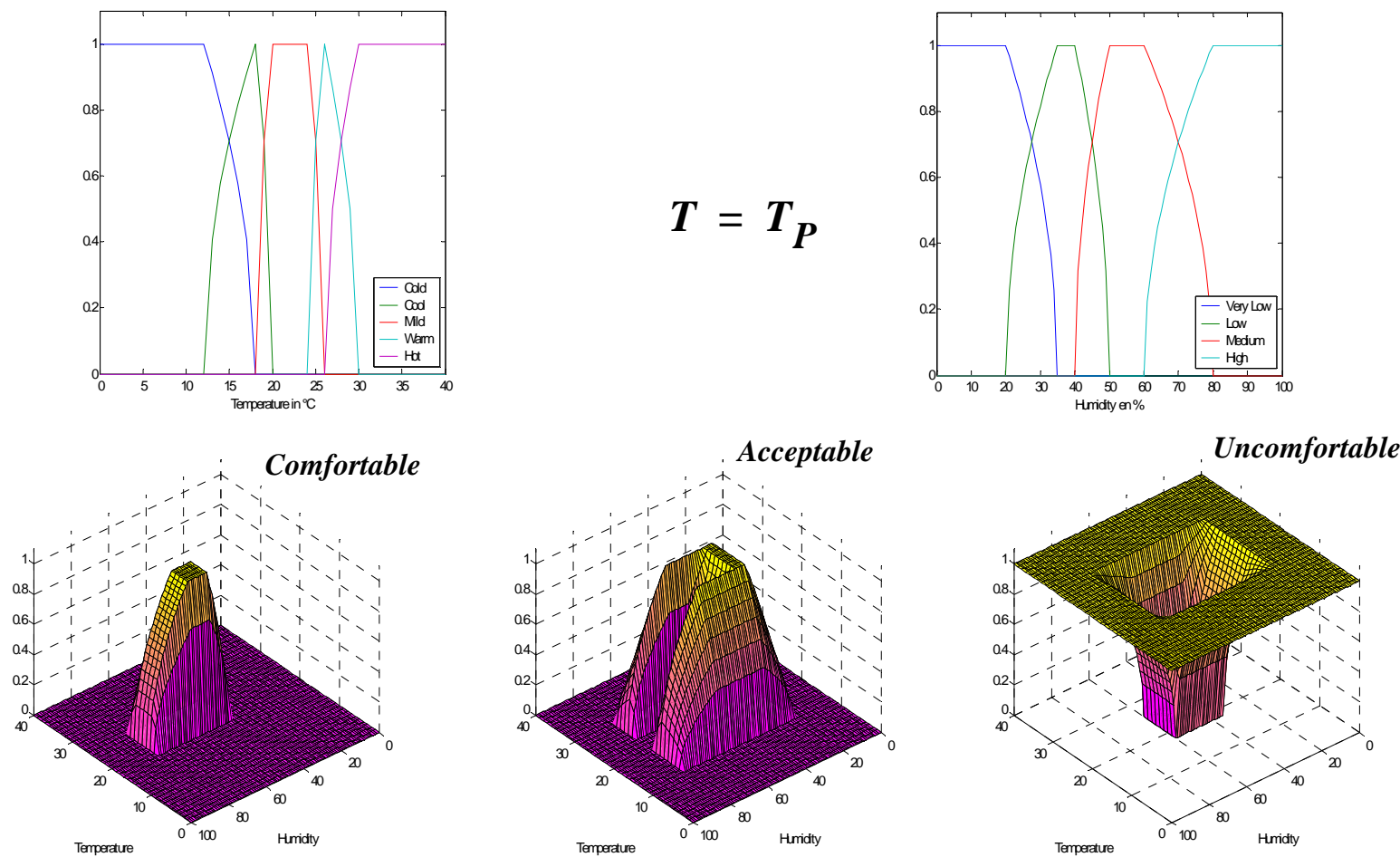

Fig. 10 Fuzzy meanings on $X_{1}, X_{2}$ and $X_{1} \times X_{2}$ for $T=T_{P}$.

The fuzzy meanings on $X_{1} \times X_{2}$ can be used to compute the fuzzy descriptions of the four situations. It leads to the table 14 .

\begin{tabular}{cccc}
\hline Situation & Comfortable & Acceptable & Uncomfortable \\
\hline$S_{1}$ & 0.000000 & 0.790569 & 0.612372 \\
$S_{2}$ & 0.000000 & 0.577350 & 0.816497 \\
$S_{3}$ & 0.707107 & 0.707107 & 0.000000 \\
$S_{4}$ & 1.000000 & 0.000000 & 0.000000 \\
\hline
\end{tabular}

Table 14: Fuzzy descriptions of the four comfort situations.

Finally, the $R_{T_{P}}$ fuzzy relation obtained is a $T_{0.5}^{Y}$-equivalence. 


\begin{tabular}{|c||c|c|c|c|}
\hline$R_{\Sigma-T_{P}}$ & $\boldsymbol{S}_{1}$ & $\boldsymbol{S}_{2}$ & $\boldsymbol{S}_{3}$ & $\boldsymbol{S}_{4}$ \\
\hline \hline $\boldsymbol{S}_{1}$ & 1.000 & 0.956 & 0.559 & 0.000 \\
\hline $\boldsymbol{S}_{2}$ & 0.956 & 1.000 & 0.408 & 0.000 \\
\hline $\boldsymbol{S}_{3}$ & 0.559 & 0.408 & 1.000 & 0.707 \\
\hline $\boldsymbol{S}_{4}$ & 0.000 & 0.000 & 0.707 & 1.000 \\
\hline
\end{tabular}

Fig. $11 R_{T_{P}}$ fuzzy equivalence relation.

\section{Conclusion}

In this paper, $R_{T}$ fuzzy relations have been introduced. It was shown that $R_{T}$ fuzzy relations are at least $T_{D^{-}}$ equivalences when $T$ is a strict t-norm with a convex additive generator. Finding the greatest $T_{\#}$ t-norm such that $R_{T}$ fuzzy relations are $T_{\#}$-equivalences is still an open question.

Several examples as close as possible to real problems were proposed to illustrate the interest of this work. For example, it justifies the choice of the operators «sum» and «product» in many rule-based applications. Indeed, when the membership functions define a strict partitioning, the sum and the product are respectively the $S^{*}$ and $T^{*}$ operators when $T=T_{M}$ since $\phi(u)=u$. Therefore, it makes it possible to have $R_{T_{M}}$ relations on the cartesian product and therefore, to preserve the associated pseudo-metric.

\section{Acknowledgements}

The authors would like to thank the referees for their valuable comments and Prof. P. Baras and R. David, LAMA, Université de Savoie, for fruitful discussions.

\section{References}

[1] E. Benoit, G. Mauris, L. Foulloy, A fuzzy colour sensor, Proc. of XIII IMEKO World congress, Torino, Italy, September 1994, pp. 1015-1020.

[2] E. Benoit, G. Mauris, L. Foulloy, Fuzzy sensor aggregation: application to comfort measurement, 5th Int. Conf. on Information Processing and Management of Uncertainty in knowledge-based systems (IPMU'94), Paris, France, July 1994, pp. 721-726.

[3] J.C. Bezdek, J.D. Harris, Fuzzy Partitions and Relations: an Axiomatic basis for Clustering, Fuzzy Sets and Systems, 1, 1978, pp. 111-127. 
[4] U. Bodenhofer, A note on approximate equality versus Poincaré paradox, Fuzzy Sets and Systems, 133(2), 2003, pp. 155-160.

[5] D. Boixader, On the relationship between $T$-transistivity and approximate equality, Fuzzy Sets and Systems, 133(2), 2003, pp. 161-169.

[6] D. Boixader, J. Jacas, J. Racacens, Fuzzy Equivalence Relations: advanced material, in Fundamentals of Fuzzy Sets, Eds. Dubois D. \& Prade H., ISBN 0-7923-7732-X, pp. 261-290.

[7] T. Calvo, B. De Baets, J. Fodor, The functional equations of Frank and Alsina for uninorms and nullnorms, Fuzzy Sets and Systems, 120, 2001, pp. 385-394.

[8] B. De Baets, R. Mesiar, Fuzzy Partitions and their Entropy, Proc. of the 6th Int. Conf. on Information Processing and Management of Uncertainty (IPMU’96), Granada, Spain, Vol. III, 1996, 1419-1424.

[9] B. De Baets, R. Mesiar, T-partitions, T-equivalences and pseudo-metrics, Proc. of the 7th Int. Fuzzy Systems Association World Congress (IFSA’97), Prage, Czech Republic, Vol. I, 1997, pp.187-192.

[10] B. De Baets, R. Mesiar, Metrics and T-Equalities, Journal of Mathematical Analysis and Applications, 267, 2002, pp. 531-547.

[11] M. De Cock, E.Kerre, A context-based approach to linguistic hedges, Int. Journal of Applied Mathematics and Computer Science, 12(3), 2002, pp. 371-382.

[12] M. De Cock, E.Kerre, On (un)suitable fuzzy relations to model approximate equality, Fuzzy Sets and Systems, 133(2), 2003, pp. 137-153.

[13] M. De Cook, E. Kerre, Why T-equivalence relations do not resolve the Poincaré paradox, and related issues, Fuzzy Sets and Systems, 133(2), 2003, pp. 181-192.

[14] A. De Soto, J. Recasens, Modelling a linguistic variable as a hierarchical family of partitions induced by an indistinguishability operator, Fuzzy Sets and Systems, 121, 2001, pp. 427-437.

[15] D. Dubois, H. Prade, Fuzzy numbers: an overview, In Analysis of Fuzzy Information vol. 1: Mathematics and Logic (J. Bezdek, Ed.), CRC Press, 1987.

[16] D. Dubois, L. Foulloy , G. Mauris, H. Prade, Probability-possibility transformations, triangular fuzzy sets and probabilistic inequalities, International Journal on Reliable Computing, Vol. 10, No. 4, 2004, pp. 273297. 
[17] L. Finkelstein, Representation by symbol systems as an extension of the concept of measurement, Kybernetes, Vol. 4, pp. 215-223, 1975.

[18] E.P. Klement, R. Mesiar, E. Pap, Triangular Norms, Trends in Logic, Vol. 8, Kluwer Academic Publishers, 2000.

[19] W. Janis, Resemblance in a nearness, Fuzzy Sets and Systems, 133(2), 2003, pp. 171-173.

[20] F. Klawonn, Should fuzzy equality and similarity satisfy transitivity? Comments on the paper by M. De Cook and E. Kerre, Fuzzy Sets and Systems, 133(2), 2003, pp. 175-180.

[21] G. Mauris, E. Benoit, L. Foulloy, Fuzzy symbolic sensor : from concept to applications, Measurement, ISSN 0263-2241, $\mathrm{N}^{\mathrm{0}}$ 12, pp 357-184, 1994.

[22] S. Ovchinnikov, An introduction to fuzzy relations, in Fundamentals of Fuzzy Sets, Eds. Dubois D. \& Prade H., ISBN 0-7923-7732-X, pp. 233-259.

[23] W. Pedricz, Why triangular membership functions?, Fuzzy Sets and Systems, No 64, 1994, pp. 21-30.

[24] W. Pedrycz, Fuzzy equalization in construction of fuzzy sets, Fuzzy Sets dand Systems, No 119, 2001, pp. 329-335.

[25] E. Ruspini, A new approach to clustering, Inform. Control, Vol. 15, No 1, 1969, pp. 22-32.

[26] E. Trillas, L. Valverde, An inquiry on indistinguishability operators, in H.J. Skala et al. (Eds.): Aspects of Vagueness, Reidel, Dordrecht, 1984, pp. 231-256.

[27] L. Valverde, On the Structure of F-indistinguishability Operators, Fuzzy Sets and Systems, 17, 1985, pp. 313328.

[28] L.A. Zadeh, Quantitative fuzzy semantics, Information Sciences, Vol. 3, 1971, pp. 159-176.

[29] L.A. Zadeh, Outline of a new approach to the analysis of complex systems and decision processes, IEEE Trans. on Systems, Man and Cybernetics, 1973, vol. SMC3, no 1, pp. 28-44. 